\title{
STOPPING CRITERIA FOR THE ITERATIVE SOLUTION OF LINEAR LEAST SQUARES PROBLEMS*
}

\author{
X.-W. CHANG ${ }^{\dagger}$, C. C. PAIGE ${ }^{\dagger}$, AND D. TITLEY-PELOQUIN ${ }^{\dagger}$
}

\begin{abstract}
We explain an interesting property of minimum residual iterative methods for the solution of the linear least squares (LS) problem. Our analysis demonstrates that the stopping criteria commonly used with these methods can in some situations be too conservative, causing any chosen method to perform too many iterations or even fail to detect that an acceptable iterate has been obtained. We propose a less conservative criterion to determine whether a given iterate is an acceptable LS solution. This is merely a sufficient condition, but it approaches a necessary condition in the limit as the given iterate approaches the exact LS solution. We also propose a necessary and sufficient condition to determine whether a given approximate LS solution is an acceptable LS solution, based on recent results on backward perturbation analysis of the LS problem. Although both of the above new conditions use quantities that are too expensive to compute in practical situations, we suggest potential approaches for estimating some of these quantities efficiently. We illustrate our results with several numerical examples.
\end{abstract}

Key words. linear least squares, iterative methods, large sparse matrix problems, stopping criteria, backward perturbation analysis

AMS subject classifications. $65 \mathrm{~F} 10,65 \mathrm{~F} 20,65 \mathrm{~F} 50,65 \mathrm{G} 50$

DOI. $10.1137 / 080724071$

1. Introduction. Given $A \in \mathbb{R}^{m \times n}$ and $b \in \mathbb{R}^{m}$, the linear least squares (LS) problem is

$$
\min _{x}\|b-A x\|_{2} .
$$

See, for example, [2] and [6] for useful background. We assume throughout that $A$ has full column rank. Under this assumption, $\hat{x}$ is the unique solution of (1.1) if and only if $A^{T}(b-A \hat{x})=0$.

In this paper we discuss stopping criteria for the iterative solution of large sparse LS problems. To make the exposition concrete we concentrate on the widely used algorithm LSQR of Paige and Saunders [12, 13]. Note, however, that much of our discussion, and our stopping criteria, are applicable to other iterative methods for the solution of large sparse LS problems. (The practical implementations of these stopping criteria will of course vary from method to method.)

In section 2 we define what we mean by an acceptable $L S$ solution and a backward stable LS solution. In section 3 we briefly summarize algorithm LSQR and state the stopping criteria originally proposed for LSQR in [12, section 5]. These are based on sufficient (but not necessary) conditions for a given iterate to be an acceptable LS solution. Note that LSQR's stopping criteria are also frequently used with other

*Received by the editors May 13, 2008; accepted for publication (in revised form) by M. H. Gutknecht May 14, 2009; published electronically July 30, 2009.

http://www.siam.org/journals/simax/31-2/72407.html

${ }^{\dagger}$ School of Computer Science, McGill University, 3480 University Street, McConnell Engineering Building, Room 318, Montreal, QC, H3A 2A7, Canada (chang@cs.mcgill.ca, paige@cs.mcgill.ca, dtitle@cs.mcgill.ca). The research of the first author was supported by NSERC of Canada grant RGPIN217191-07. The research of the second author was supported by NSERC of Canada grant RGPIN9236. The research of the third author was supported by NSERC of Canada PGS-D Fellowship. 
iterative methods for the solution of large sparse LS problems; see, for example, [4, sections 2.4 and 3.3].

In section 4 we analyze these stopping criteria. We explain an interesting property of minimum residual iterative methods and use this to show that LSQR's stopping criteria can in some situations be too conservative. The use of these criteria can cause any chosen iterative method to perform too many iterations, or in the worst case, to fail to detect that an acceptable iterate has been obtained.

In sections 5 and 6 we propose two conditions to determine whether a given iterate $x_{k} \in \mathbb{R}^{n}$ is an acceptable LS solution. The condition in section 5 is merely sufficient, but it approaches a necessary condition in the limit as $x_{k}$ approaches the exact LS solution $\hat{x}$. The condition in section 6 is both necessary and sufficient. To our knowledge, the latter new result is the only known such condition.

We give some numerical examples in section 7 in which we compare these conditions with LSQR's stopping criteria. Section 8 contains our discussion and conclusions.

1.1. Notation. We generally use upper-case letters for matrices, lower-case Roman letters for vectors and indices, and lower-case Greek letters for scalars. $e_{j}$ denotes the $j$ th column of the unit matrix $I$. The true LS solution of (1.1) is denoted by $\hat{x}$ with $\hat{r} \equiv b-A \hat{x}$, whereas $x_{k}$ is used for the $k$ th iterate of an algorithm (often LSQR here) with $r_{k} \equiv b-A x_{k}$. For vectors, $\|\cdot\|$ denotes the 2-norm. For matrices we use $\|\cdot\|_{2}$ and $\|\cdot\|_{F}$ for the 2 - and Frobenius norm, respectively, while $\|\cdot\|_{2, F}$ denotes the use of either (consistently within an expression). We use $\mathcal{R}(A)$ to denote the range of $A$, and $P_{A}$ and $P_{A}^{\perp}$ for the orthogonal projectors onto $\mathcal{R}(A)$ and the orthogonal complement of $\mathcal{R}(A)$, respectively. Assuming that $A$ has full column rank, its MoorePenrose generalized inverse is given by $A^{\dagger}=\left(A^{T} A\right)^{-1} A^{T}$, and thus for a nonzero vector $v, v^{\dagger}=v^{T} /\|v\|^{2}$. Finally, $\kappa_{2, F}(A) \equiv\|A\|_{2, F}\left\|A^{\dagger}\right\|_{2, F}$.

For the reader's convenience we give a reference table of the important quantities used in the stopping criteria discussed in this paper, together with the first equation number where each appears, and an indication of its use, or what it is.

$\begin{array}{lll}\xi_{2, F}\left(x_{k}, \alpha, \beta\right) & (2.5) & \text { (used in testing) for acceptable LS solutions } \\ \eta_{2, F}\left(x_{k}, \alpha, \beta\right) & (3.3) & \text { for acceptable nearly compatible system solutions } \\ \psi_{2, F}\left(x_{k}, \alpha, \beta\right) & (5.1) & \text { an asymptotically tight upper bound on } \xi_{2, F}\left(x_{k}, \alpha, \beta\right) \\ \omega\left(x_{k}, \theta\right) & (6.1) & \text { the minimal backward error for a compatible system } \\ \mu\left(x_{k}, \theta\right) & (6.3) & \text { the minimal backward error for a LS problem. }\end{array}$

2. Acceptable and backward stable least squares solutions. Most practical LS problems contain uncertainties in the data, and instead of solving (1.1) with ideal data $A$ and $b$, we can at best solve a nearby problem

$$
\min _{x}\|(b+f)-(A+E) x\|,
$$

where $E$ and $f$ are small in some sense. Commonly, $E$ and $f$ have small norms relative to the norms of $A$ and $b$, and we thus consider only the case

$$
\|E\|_{2, F} \leq \alpha\|A\|_{2, F} \quad \text { and } \quad\|f\| \leq \beta\|b\|
$$

for some $\alpha$ and $\beta$ satisfying $0 \leq \alpha, \beta \ll 1$ (where we hope that estimates of $\alpha$ and $\beta$ are known). In practical applications it is often the case that $\alpha \ll \beta$, because $b$ is often a vector of measurements that is subject to much larger measurement errors than the matrix $A$. 
We say that an iterate $x_{k} \in \mathbb{R}^{n}$ is an acceptable $L S$ solution when it is the exact LS solution of a problem within the accepted range of relative errors in the data. In other words, for any given $\alpha$ and $\beta$, an iterate $x_{k}$ is an acceptable LS solution if and only if there exist perturbations $E$ and $f$ satisfying

$$
(A+E)^{T}\left[b+f-(A+E) x_{k}\right]=0, \quad\|E\|_{2, F} \leq \alpha\|A\|_{2, F}, \quad\|f\| \leq \beta\|b\| .
$$

Obviously this is the case if and only if

$$
\xi_{2, F}\left(x_{k}, \alpha, \beta\right) \leq 1,
$$

where

$$
\begin{aligned}
& \xi_{2, F}\left(x_{k}, \alpha, \beta\right) \equiv \min _{E, f, \xi}\left\{\xi:(A+E)^{T}\left[b+f-(A+E) x_{k}\right]\right.=0, \\
&\left.\|E\|_{2, F} \leq \xi \alpha\|A\|_{2, F},\|f\| \leq \xi \beta\|b\|\right\} .
\end{aligned}
$$

To summarize, for any chosen $\alpha$ and $\beta$, an iterate $x_{k}$ is an acceptable LS solution if and only if it satisfies (2.4).

Even if we have perfect data $A$ and $b$, we cannot expect to solve (1.1) exactly in floating-point arithmetic. The best we can generally hope to do is to solve a problem of the form (2.1) with $\alpha=\mathcal{O}(u)$ and $\beta=\mathcal{O}(u)$ in (2.2), $u$ being the machine's unit roundoff. We say that an iterate $x_{k}$ is a backward stable $L S$ solution when it satisfies (2.4) with $\alpha=\mathcal{O}(u)$ and $\beta=\mathcal{O}(u)$. A backward stable LS solution is thus simply an acceptable LS solution with a specific choice of $\alpha$ and $\beta$.

Note that for any scalar $\tau>0$, we can verify that $\xi_{2, F}\left(x_{k}, \tau, \tau\right) \cdot \tau=\xi_{2, F}\left(x_{k}, 1,1\right)$ by using (2.5) to define $\xi_{2, F}\left(x_{k}, \tau, \tau\right) \cdot \tau$, and then replacing each quantity $\xi \tau$ in the resulting right-hand side by the new variable $\tilde{\xi}$, giving $\xi_{2, F}\left(x_{k}, 1,1\right)$. Thus

$$
\xi_{2, F}\left(x_{k}, 1,1\right) \leq \tau \quad \Leftrightarrow \quad \xi_{2, F}\left(x_{k}, \tau, \tau\right) \leq 1 .
$$

A backward stable iterate $x_{k}$ therefore satisfies $\xi_{2, F}\left(x_{k}, 1,1\right)=\mathcal{O}(u)$. Following the nomenclature in $\left[9\right.$, sections 7.1 and 20.7], we call the quantity $\xi_{2, F}\left(x_{k}, 1,1\right)$ the optimal normwise relative backward error for LS problems.

If $\xi_{2, F}\left(x_{k}, \alpha, \beta\right) \leq 1$, many known upper bounds on the relative error $\left\|\hat{x}-x_{k}\right\| /\|\hat{x}\|$ exist as a function of $\alpha$ and $\beta$; see, for example, [9, section 20.1] and [6, section 5.3.7]. Therefore, in most practical applications (but not necessarily ill-posed problems), we can be satisfied with a given $x_{k} \in \mathbb{R}^{n}$ as an approximate solution to (1.1) when it satisfies $\xi_{2, F}\left(x_{k}, \alpha, \beta\right) \leq 1$ with an appropriate choice of $\alpha$ and $\beta$.

Unfortunately, finding an analytical expression for $\xi_{2, F}\left(x_{k}, \alpha, \beta\right)$ in (2.5) remains an open question. Some easily computable upper bounds on $\xi_{2, F}\left(x_{k}, \alpha, \beta\right)$ are known, and these can be used to give sufficient conditions for $\xi_{2, F}\left(x_{k}, \alpha, \beta\right) \leq 1$. Such conditions are commonly used as stopping criteria for the iterative solution of large sparse LS problems; see, for example, [12, section 5], [4, sections 2.4 and 3.3], and [2, p. 309]. We outline some of these in the next section.

3. Algorithm LSQR and its stopping criteria. In this section we give a brief overview of algorithm LSQR $[12,13]$ and its stopping criteria. The bidiagonalization "Bidiag 2" in [12, section 3] is that given by Golub and Kahan in [5, equation (2.4)], but with the initial vector $q_{1}=A^{T} b /\left\|A^{T} b\right\|$. For LS solutions it is preferable to use the variant "Bidiag 1 " in [12, section 3], which in theory after $k$ steps produces 
matrices $U_{k+1} \in \mathbb{R}^{m \times(k+1)}$ and $V_{k} \in \mathbb{R}^{n \times k}$ such that $U_{k+1}\left(e_{1} \beta_{1}\right)=b$, where $\beta_{1} \equiv\|b\|$, and

$$
\begin{aligned}
& A V_{k}=U_{k+1} B_{k+1, k}, \quad A^{T} U_{k+1}=V_{k} B_{k+1, k}^{T}+v_{k+1} \alpha_{k+1} e_{k+1}^{T}=V_{k+1} B_{k+1}^{T}, \\
& B_{k+1, k} \equiv\left[\begin{array}{cccc}
\alpha_{1} & & & \\
\beta_{2} & \alpha_{2} & & \\
& \beta_{3} & \ddots & \\
& & \ddots & \alpha_{k} \\
& & & \beta_{k+1}
\end{array}\right] \in \mathbb{R}^{(k+1) \times k}, \quad B_{k+1} \equiv\left[B_{k+1, k} \mid e_{k+1} \alpha_{k+1}\right] .
\end{aligned}
$$

In theory $U_{k+1}$ and $V_{k}$ both have orthonormal columns, but in practice rounding errors cause a loss of orthogonality.

In the $k$ th step we find the minimum residual approximate solution of the form $x_{k}=V_{k} y_{k}$, where $y_{k} \in \mathbb{R}^{k}$ solves

$$
\min _{y}\left\|b-A V_{k} y\right\|=\min _{y}\left\|U_{k+1}\left(e_{1} \beta_{1}-B_{k+1, k} y\right)\right\|=\min _{y}\left\|e_{1} \beta_{1}-B_{k+1, k} y\right\| .
$$

Thus $x_{k}=V_{k} y_{k}$ and $r_{k} \equiv b-A x_{k}$ (for $k=1,2, \ldots$ ) are successive approximations to the true solution $\hat{x}$ and residual $\hat{r}$. The bidiagonal LS problem in (3.1) can easily be solved for $y_{k}$ using the QR factorization of $B_{k+1, k}$. A careful implementation [13] of LSQR requires only two matrix-vector products per iteration and stores only the latest columns of $U_{k+1}$ and $V_{k}$.

Listed below are the stopping criteria used in LSQR. Given an iterate $x_{k}$ with corresponding residual $r_{k} \equiv b-A x_{k}$, the algorithm stops if one of the following three conditions is satisfied:

$$
\begin{cases}\text { 1. }\left\|r_{k}\right\| \leq \alpha\|A\|_{2, F}\left\|x_{k}\right\|+\beta\|b\| & \text { (a test for compatible systems), } \\ \text { 2. }\left\|A^{T} r_{k}\right\| /\left\|r_{k}\right\| \leq \alpha\|A\|_{2, F} & \text { (a criterion for LS problems), } \\ \text { 3. } \quad \kappa_{2, F}(A) \geq \gamma & \text { (a regularizing criterion). }\end{cases}
$$

The parameters $\alpha$ and $\beta$ (distinct from the elements $\alpha_{k}$ and $\beta_{k}$ of $B_{k+1, k}$ ) are set according to the accuracy of the data; see (2.2). From now on we assume the sensible case that $0<\alpha, \beta \ll 1$. If rough estimates of these relative errors are not known, $\alpha$ and $\beta$ could be set to a small multiple of the unit roundoff $u$. The parameter $\gamma$ is the maximum condition number we are willing to tolerate. (In LSQR, $\kappa_{F}\left(B_{k+1, k}\right)$, which is no greater than $\kappa_{F}(A)$, is checked against $\gamma$.) Note that criterion 2 in (3.2) assumes $r_{k} \neq 0$. If the residual is zero, then $x_{k}$ is clearly the exact solution $\hat{x}$ of (1.1).

LSQR provides the user with cheap estimates of $\left\|r_{k}\right\|$ and $\left\|A^{T} r_{k}\right\|$ at each iteration; see, for example, [12, section 5]. Cheap lower bounds on $\|A\|_{F}$ and $\kappa_{F}(A)$ are also available, and lower bounds on $\|A\|_{2}$ and $\kappa_{2}(A)$ can be computed reasonably cheaply at each iteration. These lower bounds are monotonically increasing with $k$ and are thus successively better approximations to $\|A\|_{2, F}$ and $\kappa_{2, F}(A)$. Similar estimates are also usually available in other iterative methods for the solution of LS problems, such as CGLS (see, for example, [2, section 7]). When using such estimates, one should keep in mind that they are not always accurate and can give misleading results. We demonstrate this with an example in section 4.3.

We now show that LSQR's stopping criteria 1 and 2 correspond to particular upper bounds on $\xi_{2, F}\left(x_{k}, \alpha, \beta\right)$ in (2.5). LSQR's stopping criteria 1 and 2 thus give sufficient but not necessary conditions for $x_{k}$ to be an acceptable LS solution. 
Criterion 1 in (3.2) can be obtained by tightening the normal equations constraint for LS in (2.5) to an equality constraint for compatible systems. Since this cannot decrease the resulting minimum value of $\xi$, it follows that

$$
\begin{aligned}
& \xi_{2, F}\left(x_{k}, \alpha, \beta\right) \leq \eta_{2, F}\left(x_{k}, \alpha, \beta\right) \\
& \quad \equiv \min _{E, f, \eta}\left\{\eta:(A+E) x_{k}=b+f,\|E\|_{2, F} \leq \eta \alpha\|A\|_{2, F},\|f\| \leq \eta \beta\|b\|\right\} .
\end{aligned}
$$

Rigal and Gaches [14] showed that

$$
\eta_{2, F}\left(x_{k}, \alpha, \beta\right)=\frac{\left\|r_{k}\right\|}{\alpha\|A\|_{2, F}\left\|x_{k}\right\|+\beta\|b\|} .
$$

They proposed the condition $\eta_{2, F}\left(x_{k}, \alpha, \beta\right) \leq 1$, in other words,

$$
\left\|r_{k}\right\| \leq \alpha\|A\|_{2, F}\left\|x_{k}\right\|+\beta\|b\|
$$

as a stopping criterion for the iterative solution of compatible linear systems. Note that for any scalar $\tau>0$,

$$
\eta_{2, F}\left(x_{k}, \tau, \tau\right) \leq 1 \quad \Leftrightarrow \quad \eta_{2, F}\left(x_{k}, 1,1\right) \leq \tau .
$$

The quantity

$$
\eta_{2, F}\left(x_{k}, 1,1\right) \equiv \frac{\left\|r_{k}\right\|}{\|A\|_{2, F}\left\|x_{k}\right\|+\|b\|}
$$

is known in the literature as the optimal normwise relative backward error for compatible systems; see [9, Theorem 7.1].

Since the LS method is often used for solving nearly compatible overdetermined linear systems, the condition (3.5) can be used as a stopping criterion for the iterative solution of LS problems, hence criterion 1 in (3.2). From (3.3) and (3.4), if (3.5) holds, then $\xi_{2, F}\left(x_{k}, \alpha, \beta\right) \leq 1$, and the iterate $x_{k}$ is an acceptable LS solution. Similarly, if $\eta_{2, F}\left(x_{k}, 1,1\right)=\mathcal{O}(u)$, then $\xi_{2, F}\left(x_{k}, 1,1\right)=\mathcal{O}(u)$, and $x_{k}$ is a backward stable LS solution; see (2.6).

Criterion 2 in (3.2) can be obtained from the fact that any feasible perturbations $E$ and $f$ in (2.5) must lead to an upper bound on the minimum $\xi_{2, F}\left(x_{k}, \alpha, \beta\right)$. Stewart [16, section 3] observed that the perturbations $E_{0}=-r_{k} r_{k}^{\dagger} A$ and $f_{0}=0$ satisfy the normal equations constraint in (2.5). With $E=E_{0}$ and $f=f_{0},(2.5)$ gives

$$
\xi_{2, F}\left(x_{k}, \alpha, \beta\right) \leq \min _{\xi}\left\{\xi:\left\|E_{0}\right\|_{2, F} \leq \xi \alpha\|A\|_{2, F}\right\}=\frac{\left\|E_{0}\right\|_{2, F}}{\alpha\|A\|_{2, F}}=\frac{\left\|A^{T} r_{k}\right\|}{\alpha\|A\|_{2, F}\left\|r_{k}\right\|} .
$$

Therefore if criterion 2 in (3.2) is satisfied, then $\xi_{2, F}\left(x_{k}, \alpha, \beta\right) \leq 1$, and, from (2.4), $x_{k}$ is an acceptable LS solution. Furthermore, if $\left\|A^{T} r_{k}\right\| /\left\|r_{k}\right\|=\mathcal{O}(u)\|A\|_{2, F}$, then $\xi_{2, F}\left(x_{k}, 1,1\right)=\mathcal{O}(u)$, and $x_{k}$ is a backward stable LS solution - see the line following (2.6). Note that $A^{T} r_{k}$ is the residual vector of the normal equations at $x_{k}$, and the quantity $\left\|A^{T} r_{k}\right\| /\left\|r_{k}\right\|$ is the norm of a backward perturbation matrix in $A$ only.

Criterion 3 in (3.2) tells us to stop if our "reduced representation" (3.1) of the problem becomes too ill-conditioned; it is an attempt to regularize ill-conditioned problems. As the focus of this paper is not on regularization of ill-conditioned problems, we will not discuss criterion 3 further.

In the next section we give new insights into the behavior of the quantities $\left\|r_{k}\right\|$ and $\left\|A^{T} r_{k}\right\| /\left\|r_{k}\right\|$ that are used in LSQR's stopping criteria 1 and 2 in (3.2). 


\section{Analysis of LSQR's stopping criteria 1 and 2.}

4.1. An interesting observation. On all problems we have tested we have made the following observation:

LSQR first reduces the residual norm $\left\|r_{k}\right\|$, while $\left\|A^{T} r_{k}\right\| /\left\|r_{k}\right\|$ remains roughly constant or tends to oscillate in ill-conditioned problems. The residual norm $\left\|r_{k}\right\|$ then reaches a plateau (after which it remains almost constant), at which point the quantity $\left\|A^{T} r_{k}\right\| /\left\|r_{k}\right\|$ starts to decrease, and decreases until it too reaches a plateau.

This surprising behavior, for which we propose an explanation below, is clearly illustrated in Figure 1 (repeated in more detail as "Test Problem 3" in Figure 3).

The oscillation of $\left\|A^{T} r_{k}\right\|$ has often been observed in various iterative methods for the solution of large sparse LS problems. For example, it is stated in [12, section $6.2]$ that in practice in LSQR " $\left\|A^{T} r_{k}\right\| /\left\|r_{k}\right\|$ can vary rather dramatically with $k$, but it does tend to stabilize for large $k . "$ Björck [2, p. 289] states that in CGLS " $\left\|A^{T} r_{k}\right\|$ will often exhibit large oscillations when $\kappa(A)$ is large." In [4] Choi uses MINRES (see [11, section 6]) and a new variant thereof to solve singular symmetric linear systems. Since these have either no solution or infinitely many solutions, they can be solved as (possibly compatible, rank-deficient) LS problems. Like LSQR but unlike MINRES, algorithm MINRES-QLP given in [4] converges to the minimum 2-norm LS solution. It is remarked [4, p. 27] that $\left\|A^{T} r_{k}\right\|$ "is often observed to be oscillating."

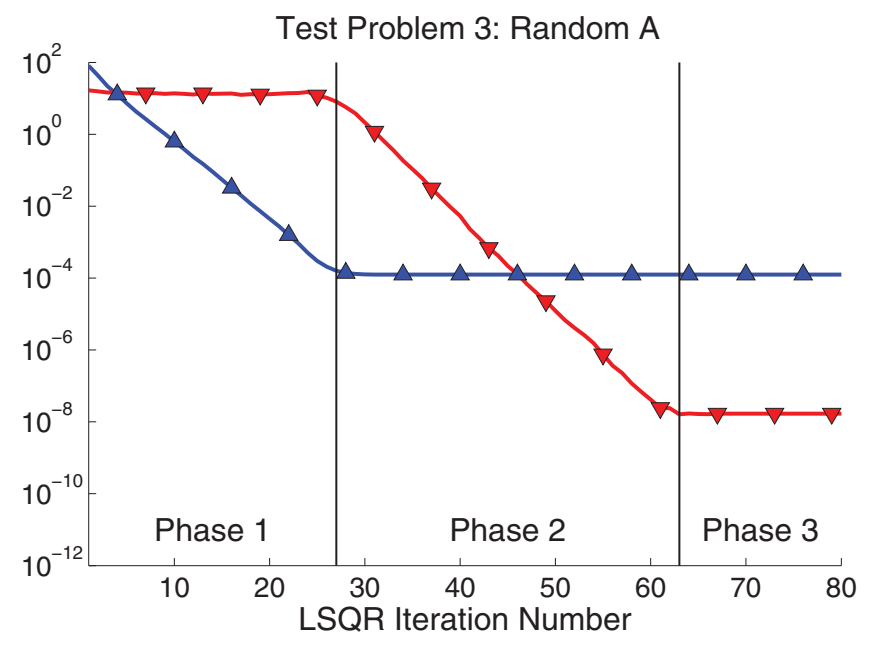

FiG. 1. Behavior of $\left\|r_{k}\right\|$ ( $\left.\mathbf{\Delta}\right)$ and $\left\|A^{T} r_{k}\right\| /\left\|r_{k}\right\|$ ( $\left.\mathbf{v}\right)$.

4.2. A possible explanation. Assume that $m \times n A$ has full column rank, and let the singular value decomposition (SVD) of $A$ be

$$
A=U\left[\begin{array}{c}
\Sigma \\
0
\end{array}\right] V^{T}=U_{1} \Sigma V^{T}=\sum_{i=1}^{n} u_{i} \sigma_{i} v_{i}^{T},
$$

where $U \equiv\left[\begin{array}{ll}U_{1} & U_{2}\end{array}\right] \equiv\left[u_{1}, \ldots, u_{m}\right] \in \mathbb{R}^{m \times m}$ and $V \equiv\left[v_{1}, \ldots, v_{n}\right] \in \mathbb{R}^{n \times n}$ (distinct from $U_{k}$ and $V_{k}$ in section 3$)$ are orthogonal matrices and $\Sigma=\operatorname{diag}\left(\sigma_{1}, \ldots, \sigma_{n}\right)$ with $\sigma_{1} \geq \cdots \geq \sigma_{n}>0$. With this notation, the orthogonal projectors onto the range of $A$ and onto the orthogonal complement of the range of $A$ are, respectively,

$$
P_{A} \equiv U_{1} U_{1}^{T} \quad \text { and } \quad P_{A}^{\perp} \equiv U_{2} U_{2}^{T} .
$$


If $\hat{x}$ is the true LS solution with corresponding residual $\hat{r} \equiv b-A \hat{x}$, then

$$
P_{A}^{\perp} r_{k}=U_{2} U_{2}^{T}\left(b-A x_{k}\right)=U_{2} U_{2}^{T} b=P_{A}^{\perp} b=\hat{r}
$$

for all $k$, so that

$$
\left\|r_{k}\right\|^{2}=\left\|P_{A} r_{k}\right\|^{2}+\left\|P_{A}^{\perp} r_{k}\right\|^{2}=\left\|P_{A} r_{k}\right\|^{2}+\|\hat{r}\|^{2} .
$$

Note that in theory LSQR decreases $\left\|r_{k}\right\|$ every step (see [12, equation (5.2) and p. 50]) so it also decreases $\left\|P_{A} r_{k}\right\|$ every step. Furthermore, because $V_{n}$ in section 3 is theoretically orthogonal, in theory $\left\|P_{A} r_{k}\right\|=0$ when $k=n$ (and possibly even for some $k<n$ ). Thus in LSQR $\left\|P_{A} r_{k}\right\|$ converges strictly monotonically to 0 . We need the following lemma.

Lemma 4.1. Given $A \in \mathbb{R}^{m \times n}$ with the above-defined $S V D, b \in \mathbb{R}^{m}$, and $x_{k} \in \mathbb{R}^{n}$, define $r_{k} \equiv b-A x_{k}$. Then

$$
\left\|A^{T} r_{k}\right\|=\bar{\sigma}_{k}\left\|P_{A} r_{k}\right\|
$$

for some $\bar{\sigma}_{k}$ in the closed interval $\left[\sigma_{n}, \sigma_{1}\right]$.

Proof. Using the SVD of $A$,

$$
\left\|A^{T} r_{k}\right\|^{2}=r_{k}^{T} U_{1} \Sigma^{2} U_{1}^{T} r_{k}=\sum_{i=1}^{n}\left(u_{i}^{T} r_{k}\right)^{2} \sigma_{i}^{2}=\bar{\sigma}_{k}^{2} \sum_{i=1}^{n}\left(u_{i}^{T} r_{k}\right)^{2}
$$

for some $\bar{\sigma}_{k} \in\left[\sigma_{n}, \sigma_{1}\right]$. Now because

$$
\left\|P_{A} r_{k}\right\|^{2}=r_{k}^{T} U_{1} U_{1}^{T} r_{k}=\sum_{i=1}^{n}\left(u_{i}^{T} r_{k}\right)^{2},
$$

it immediately follows that $\left\|A^{T} r_{k}\right\|=\bar{\sigma}_{k}\left\|P_{A} r_{k}\right\|$.

In well-conditioned problems the singular values of $A$ are all very roughly of similar orders of magnitude. Therefore in well-conditioned problems the order of magnitude of $\bar{\sigma}_{k}$ is very roughly constant as a function of $k$. In ill-conditioned problems, $\bar{\sigma}_{k}$ can oscillate wildly but always lies between the extreme singular values of $A$. Note that the behavior of $\bar{\sigma}_{k}$ as a function of $k$ depends on the size of the residual norms and on how the residuals are aligned with respect to the left singular vectors $u_{1}$ to $u_{n}$.

We can now describe what appears to be the main basis for the interesting observation. We do so by dividing the LSQR iteration process into three phases, illustrated in Figure 1, any of which need not exist.

Phase 1. This phase is defined by those iterates for which in (4.2)

$$
\left\|P_{A} r_{k}\right\|>\left\|P_{A}^{\perp} r_{k}\right\|=\|\hat{r}\|,
$$

and so from (4.2),

$$
\left\|r_{k}\right\|^{2}=\left\|P_{A} r_{k}\right\|^{2}+\|\hat{r}\|^{2} \approx\left\|P_{A} r_{k}\right\|^{2} .
$$

Thus in phase $1\left\|r_{k}\right\| \approx\left\|P_{A} r_{k}\right\|$ and LSQR decreases $\left\|r_{k}\right\|$, while from (4.5) and Lemma 4.1,

$$
\frac{\left\|A^{T} r_{k}\right\|}{\left\|r_{k}\right\|}=\frac{\bar{\sigma}_{k}\left\|P_{A} r_{k}\right\|}{\left\|r_{k}\right\|} \approx \bar{\sigma}_{k}
$$

Copyright $@$ by SIAM. Unauthorized reproduction of this article is prohibited. 
which must lie between the extreme singular values of $A$. Thus in phase 1 Stewart's $\left\|A^{T} r_{k}\right\| /\left\|r_{k}\right\|$ is roughly constant in well-conditioned problems and can oscillate in ill-conditioned problems.

The sum of squares in (4.5) makes (4.6) a particularly good approximation even when $\left\|P_{A} r_{k}\right\|$ is not very much larger than $\left\|P_{A}^{\perp} r_{k}\right\|=\|\hat{r}\|$. For example if $\left\|P_{A} r_{k}\right\|=$ $2\|\hat{r}\|$, we get $\left\|r_{k}\right\|=(\sqrt{5} / 2)\left\|P_{A} r_{k}\right\|$, leading to a relative error of only $\left(\left\|r_{k}\right\|-\right.$ $\left.\left\|P_{A} r_{k}\right\|\right) /\left\|P_{A} r_{k}\right\| \approx 12 \%$. If $\left\|P_{A} r_{k}\right\|=10\|\hat{r}\|$, the relative error becomes approximately $0.5 \%$.

Usually the iteration starts in phase 1 , because usually $\left\|r_{0}\right\| \gg\|\hat{r}\|$ and thus $\left\|P_{A} r_{0}\right\| \gg\|\hat{r}\|$; see (4.5) with $k=0$. However, it may happen that $\left\|P_{A} r_{0}\right\| \leq\|\hat{r}\|$, for example, if $x_{0}$ is a very good approximation to $\hat{x}$. In this case there is no phase 1 and the iteration starts in either phase 2 or phase 3 below.

Phase 2. First suppose that the linear system is not compatible to machine precision. We consider the compatible case afterwards.

As LSQR decreases $\left\|P_{A} r_{k}\right\|$, there is a first $k$ such that

$$
\left\|P_{A} r_{k}\right\| \leq\left\|P_{A}^{\perp} r_{k}\right\|=\|\hat{r}\| ;
$$

see (4.1) and (4.2). This is the start of phase 2, in which the residuals are now dominated by their projection onto the orthogonal complement of $\mathcal{R}(A)$. In this phase $\left\|P_{A} r_{k}\right\|$ continues to decrease but $\left\|r_{k}\right\|$ hardly decreases because it is dominated by $\left\|P_{A}^{\perp} r_{k}\right\|=\|\hat{r}\|$, which is constant.

Because $\left\|P_{A} r_{k}\right\|$ still decreases while $\left\|r_{k}\right\|$ remains roughly constant, from (4.3) the quantity $\left\|A^{T} r_{k}\right\| /\left\|r_{k}\right\|=\bar{\sigma}_{k}\left\|P_{A} r_{k}\right\| /\left\|r_{k}\right\|$ tends to decrease. Thus in phase 2 it is $\left\|r_{k}\right\|$ that remains nearly constant, while $\left\|A^{T} r_{k}\right\| /\left\|r_{k}\right\|$ tends to decrease.

As LSQR continues to decrease $\left\|P_{A} r_{k}\right\|$ (recall that $\left\|P_{A} r_{k}\right\| \rightarrow 0$ in theory), there is a first $k$ such that

$$
\left\|P_{A} r_{k}\right\|=\mathcal{O}(u)\left(\|A\|_{2, F}\left\|x_{k}\right\|+\|b\|\right) .
$$

This implies that $x_{k}$ is a backward stable LS solution; see section 5 for a detailed explanation.

Phase 3. This phase begins when LSQR has decreased $\left\|P_{A} r_{k}\right\|$ to the level in (4.8). In all our numerical experiments we have found that $\left\|P_{A} r_{k}\right\|$ does not decrease below this level, even though in theory $\left\|P_{A} r_{k}\right\| \rightarrow 0$. This is true even for compatible systems where in theory $\left\|r_{k}\right\| \rightarrow 0$. The numerical examples in section 7 illustrate this behavior.

Now suppose that the linear system is compatible to the level of machine precision, meaning that the true LS solution $\hat{x}$ satisfies $\eta_{2, F}(\hat{x}, 1,1)=\mathcal{O}(u)$; see (3.3) and (3.6). In this case, in apparently all but the most extreme circumstances, LSQR converges to an iterate $x_{k}$ that also solves $A x=b$ to the level of machine precision. In other words, for these problems there is usually a $k$ such that $\eta_{2, F}\left(x_{k}, 1,1\right)=\mathcal{O}(u)$ and thus

$$
\left\|r_{k}\right\|=\mathcal{O}(u)\left(\|A\|_{2, F}\left\|x_{k}\right\|+\|b\|\right)
$$

see (3.4) and (3.6). In this case there is effectively no phase 2 (and phase 3 starts immediately after phase 1 ) because if (4.9) is satisfied, then clearly so is (4.8).

In phase 3 neither $\left\|r_{k}\right\|$ nor $\left\|A^{T} r_{k}\right\| /\left\|r_{k}\right\|$ decreases further. If the system is not compatible to machine precision, then as in phase $2\left\|r_{k}\right\|$ is dominated by $\left\|P_{A}^{\perp} r_{k}\right\|=$ $\|\hat{r}\|$, which is constant. If the linear system is compatible to machine precision, then $\left\|r_{k}\right\|$ satisfies (4.9) and is therefore roughly constant. Thus we have

$$
\left\|r_{k}\right\| \approx \max \left\{\|\hat{r}\|, \mathcal{O}(u)\left(\|A\|_{2, F}\left\|x_{k}\right\|+\|b\|\right)\right\}
$$


and from (4.3) and (4.8) we obtain

$$
\frac{\left\|A^{T} r_{k}\right\|}{\left\|r_{k}\right\|}=\frac{\bar{\sigma}_{k}\left\|P_{A} r_{k}\right\|}{\left\|r_{k}\right\|}=\bar{\sigma}_{k} \frac{\mathcal{O}(u)\left(\|A\|_{2, F}\left\|x_{k}\right\|+\|b\|\right)}{\left\|r_{k}\right\|} .
$$

In phase 3 the ratio $\bar{\sigma}_{k}$ usually remains almost constant (even for ill-conditioned problems; see the convergence plots in Figure 4). This is due to $r_{k}$ being nearly constant in this phase, so from Lemma $4.1 \bar{\sigma}_{k}$ is also almost constant. Therefore $\left\|A^{T} r_{k}\right\| /\left\|r_{k}\right\|$ also remains nearly constant.

Summary. In summary, initially $\left\|A^{T} r_{k}\right\| /\left\|r_{k}\right\| \approx \bar{\sigma}_{k} \in\left[\sigma_{n}, \sigma_{1}\right]$ is roughly constant in well-conditioned problems and oscillates between the extreme singular values of $A$ in ill-conditioned problems. This quantity starts to decrease only once $\left\|r_{k}\right\|$ is no longer dominated by $\left\|P_{A} r_{k}\right\|$, which happens when $\left\|r_{k}\right\|$ reaches the plateau in (4.10). Eventually $\left\|A^{T} r_{k}\right\| /\left\|r_{k}\right\|$ also reaches the plateau in (4.11), where it too remains almost constant.

4.3. Relation to the stopping criteria 1 and 2 . We can relate the above observations to the stopping criteria 1 and 2 in (3.2), which give sufficient but not necessary conditions for $x_{k}$ to be an acceptable LS solution. Since the theoretically strictly monotonically decreasing $\left\|r_{k}\right\|$ no longer decreases significantly after phase 1 and plateaus at the level given in (4.10), LSQR's criterion 1 may never be triggered in significant-residual problems (for which the maximum in (4.10) is given by $\|\hat{r}\|$ ). On the other hand, because $\left\|A^{T} r_{k}\right\| /\left\|r_{k}\right\|$ reaches a plateau (4.11) at the start of phase 3 , LSQR's criterion 2 may never be triggered in nearly compatible systems (for which (4.9) holds in phase 3 and (4.11) is thus roughly of the order of $\bar{\sigma}_{k}$ ). So both stopping criteria are needed - but even then there can be difficulties.

We illustrate this with an example in Figure 2 (repeated with different detail as "Test Problem 3" in Figure 3). Suppose that we would like to obtain a backward stable LS solution, so we set $\alpha=\beta=u$ in (2.5) and (3.2). It is easy to see from the plotted tolerances in Figure 2 that neither stopping criterion in (3.2) is ever triggered, regardless of the number of iterations performed. This demonstrates that LSQR's stopping criteria can be much too conservative, and can lead a user to incorrect conclusions about whether or not LSQR (or any other algorithm) has converged to a required tolerance.

In the next section we give a new tighter estimate of $\xi_{2, F}\left(x_{k}, \alpha, \beta\right)$. This result indicates that in the above example in fact $\xi_{2}\left(x_{k}, 1,1\right)=\mathcal{O}(u)$ (see $(2.6)$ ) when $k=63$. In other words, a backward stable iterate (in the 2-norm) is obtained at the end of phase 2 .

We note that although the quantity $\left\|A^{T} r_{k}\right\| /\left\|r_{k}\right\|$ plateaus in what we have called phase 3, in practice LSQR's approximation to $\left\|A^{T} r_{k}\right\| /\left\|r_{k}\right\|$ generally does not, as shown in Figure 2. Therefore stopping criterion 2 in (3.2) may be triggered if we use LSQR's approximation in our computation, even though the actual $\left\|A^{T} r_{k}\right\| /\left\|r_{k}\right\| \gg$ $\alpha\|A\|_{2, F}$. In the above example this leads to stopping criterion 2 (in the 2-norm with $\alpha=u$ ) being triggered at the iteration $k=83$, late in phase 3 and well after a backward stable iterate has actually been obtained.

5. A new upper bound on $\xi_{2, F}\left(x_{k}, \alpha, \beta\right)$. We now give a new upper bound on $\xi_{2, F}\left(x_{k}, \alpha, \beta\right)$ defined in (2.5), and show that it becomes asymptotically tight in the limit as $x_{k}$ approaches the true LS solution $\hat{x}$ of (1.1). This bound could be used to improve LSQR's stopping criteria significantly. 


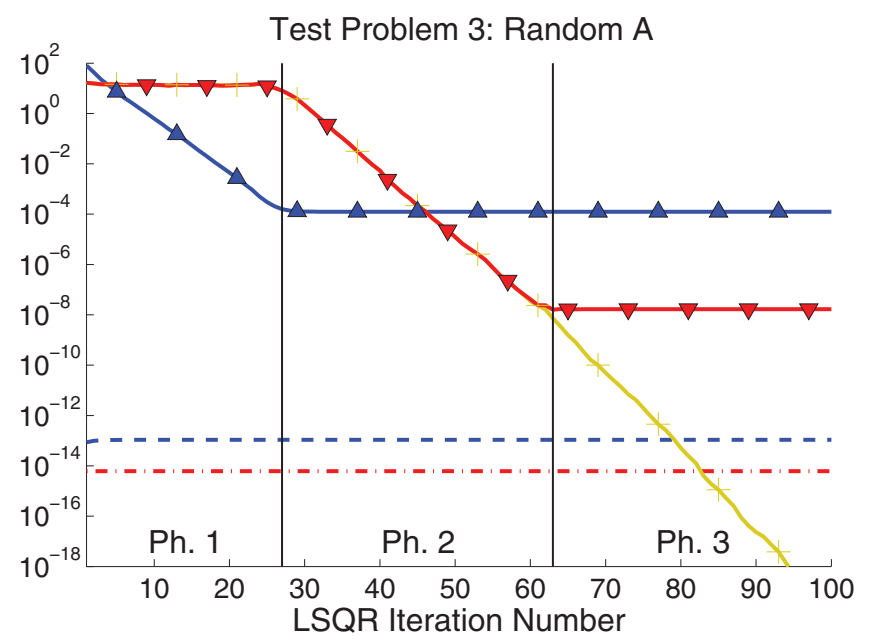

$$
\begin{array}{ll}
\longrightarrow & \begin{array}{l}
\text { residual norm }\left\|r_{k}\right\| \\
\text { Stewart's }\left\|A^{T} r_{k}\right\| /\left\|r_{k}\right\|
\end{array} \\
& \begin{array}{l}
\text { LSQR's approximation to }\left\|A^{T} r_{k}\right\| /\left\|r_{k}\right\| \\
\text { - = - - }
\end{array} \\
\text { tolerance } u\left(\|A\|_{2}\left\|x_{k}\right\|+\|b\|\right) \\
\text { tolerance } u\|A\|_{2}
\end{array}
$$

FIG. 2. Behavior of $\left\|r_{k}\right\|$ and $\left\|A^{T} r_{k}\right\| /\left\|r_{k}\right\|$.

Theorem 5.1. Given $A \in \mathbb{R}^{m \times n}, b \in \mathbb{R}^{m}$, and $x_{k} \in \mathbb{R}^{n}$, define $r_{k} \equiv b-A x_{k}$ and use the definition of $\xi_{2, F}\left(x_{k}, \alpha, \beta\right)$ in (2.5). Then

$$
\xi_{2, F}\left(x_{k}, \alpha, \beta\right) \leq \psi_{2, F}\left(x_{k}, \alpha, \beta\right) \equiv \frac{\left\|P_{A} r_{k}\right\|}{\alpha\|A\|_{2, F}\left\|x_{k}\right\|+\beta\|b\|} .
$$

Proof. Consider the perturbations

$$
\begin{aligned}
E^{*} & \equiv \frac{\alpha\|A\|_{2, F}\left\|x_{k}\right\|}{\alpha\|A\|_{2, F}\left\|x_{k}\right\|+\beta\|b\|} P_{A} r_{k} x_{k}^{\dagger}, \\
f^{*} & \equiv-\frac{\beta\|b\|}{\alpha\|A\|_{2, F}\left\|x_{k}\right\|+\beta\|b\|} P_{A} r_{k},
\end{aligned}
$$

so that

$$
\frac{\left\|E^{*}\right\|_{2, F}}{\alpha\|A\|_{2, F}}=\frac{\left\|f^{*}\right\|}{\beta\|b\|}=\frac{\left\|P_{A} r_{k}\right\|}{\alpha\|A\|_{2, F}\left\|x_{k}\right\|+\beta\|b\|}=\psi_{2, F}\left(x_{k}, \alpha, \beta\right) .
$$

Also notice that $b+f^{*}-\left(A+E^{*}\right) x_{k}=r_{k}-P_{A} r_{k}=P_{A}^{\perp} r_{k}$. From this we see that $E^{*}$ and $f^{*}$ satisfy the normal equations constraint in (2.5). It then follows from (2.5) and (5.2) that

$$
\xi_{2, F}\left(x_{k}, \alpha, \beta\right) \leq \min _{\xi}\left\{\xi:\left\|E^{*}\right\|_{2, F} \leq \xi \alpha\|A\|_{2, F},\left\|f^{*}\right\| \leq \xi \beta\|b\|\right\}=\psi_{2, F}\left(x_{k}, \alpha, \beta\right),
$$

so that (5.1) holds.

Copyright $@$ by SIAM. Unauthorized reproduction of this article is prohibited. 
Recall that an iterate $x_{k}$ is an acceptable LS solution if and only if it satisfies $\xi_{2, F}\left(x_{k}, \alpha, \beta\right) \leq 1$ (see (2.5)). As a result of Theorem 5.1, if

$$
\text { LStest1: } \quad \psi_{2, F}\left(x_{k}, \alpha, \beta\right) \leq 1
$$

or, equivalently,

$$
\left\|P_{A} r_{k}\right\| \leq \alpha\|A\|_{2, F}\left\|x_{k}\right\|+\beta\|b\|,
$$

then $\xi_{2, F}\left(x_{k}, \alpha, \beta\right) \leq 1$ and $x_{k}$ is an acceptable LS solution. Comparing the above to LSQR's stopping criterion 1 in (3.2), we immediately see that (5.4) gives a less pessimistic criterion. In fact the upper bound $\psi_{2, F}$ in (5.1) can be much tighter than $\eta_{2, F}$ in (3.3) (see also (3.4)), especially during what we have called phases 2 and 3 when $\left\|P_{A} r_{k}\right\|$ is no longer the main component of $\left\|r_{k}\right\|$.

We can use Theorem 5.1 to explain why $\left\|P_{A} r_{k}\right\|$ levels off at the end of phase 2, as illustrated in Figure 3 and noted after (4.8). If (4.8) holds, then from (5.1) we have $\xi_{2, F}\left(x_{k}, 1,1\right)=\mathcal{O}(u)$ and thus from (2.6) $x_{k}$ is a backward stable LS solution. As discussed in section 2, this is the best we can generally hope to achieve in floatingpoint arithmetic. Therefore, we cannot generally expect that $\left\|P_{A} r_{k}\right\|$ will decrease below the level given in (4.8).

We now show that our new upper bound $\psi_{2, F}\left(x_{k}, \alpha, \beta\right)$ in (5.1) becomes asymptotically tight with $\xi_{2, F}\left(x_{k}, \alpha, \beta\right)$ in (2.5) in the limit as $x_{k}$ approaches the true LS solution $\hat{x}$. Note that in theory as $x_{k} \rightarrow \hat{x}, \psi_{2, F}\left(x_{k}, \alpha, \beta\right) \rightarrow 0$ and so $\xi_{2, F}\left(x_{k}, \alpha, \beta\right) \rightarrow 0$; see (5.1). The following theorem shows that both converge at the same rate.

THEOREM 5.2. Using the notation of Theorem 5.1 and letting $\hat{x}$ denote the true $L S$ solution of (1.1),

$$
\lim _{x_{k} \rightarrow \hat{x}} \frac{\xi_{2, F}\left(x_{k}, \alpha, \beta\right)}{\psi_{2, F}\left(x_{k}, \alpha, \beta\right)}=1
$$

Proof. We have shown in Theorem 5.1 that $\xi_{2, F}\left(x_{k}, \alpha, \beta\right) \leq \psi_{2, F}\left(x_{k}, \alpha, \beta\right)$ for all $x_{k} \in \mathbb{R}^{n}$. Therefore,

$$
\lim _{x_{k} \rightarrow \hat{x}} \frac{\xi_{2, F}\left(x_{k}, \alpha, \beta\right)}{\psi_{2, F}\left(x_{k}, \alpha, \beta\right)} \leq 1
$$

On the other hand, notice that the optimal perturbations $\hat{E}_{k}$ and $\hat{f}_{k}$ in (2.5) must satisfy

$$
\left(A+\hat{E}_{k}\right)^{T}\left(r_{k}+\hat{f}_{k}-\hat{E}_{k} x_{k}\right)=0
$$

so that $P_{A+\hat{E}_{k}}\left(r_{k}+\hat{f}_{k}-\hat{E}_{k} x_{k}\right)=0$ and thus $P_{A+\hat{E}_{k}} r_{k}=P_{A+\hat{E}_{k}}\left(\hat{E}_{k} x_{k}-\hat{f}_{k}\right)$. Using the fact that $\left\|P_{A+\hat{E}_{k}}\right\| \leq 1$ along with the other constraints in (2.5), it follows that the optimal $\xi_{2, F}\left(x_{k}, \alpha, \beta\right)$ in (2.5) must satisfy

$$
\left\|P_{A+\hat{E}_{k}} r_{k}\right\| \leq\left\|\hat{E}_{k}\right\|_{2, F}\left\|x_{k}\right\|+\left\|\hat{f}_{k}\right\| \leq \xi_{2, F}\left(x_{k}, \alpha, \beta\right) \cdot\left(\alpha\|A\|_{2, F}\left\|x_{k}\right\|+\beta\|b\|\right),
$$

so that with (5.1),

$$
\xi_{2, F}\left(x_{k}, \alpha, \beta\right) \geq \frac{\left\|P_{A+\hat{E}_{k}} r_{k}\right\|}{\alpha\|A\|_{2, F}\left\|x_{k}\right\|+\beta\|b\|}=\psi_{2, F}\left(x_{k}, \alpha, \beta\right) \frac{\left\|P_{A+\hat{E}_{k}} r_{k}\right\|}{\left\|P_{A} r_{k}\right\|} .
$$

Copyright (c) by SIAM. Unauthorized reproduction of this article is prohibited. 
In the limit as $x_{k} \rightarrow \hat{x}$ we have $\left\|\hat{E}_{k}\right\|_{2, F} \rightarrow 0$ and thus $P_{A+\hat{E}_{k}} r_{k} \rightarrow P_{A} r_{k}$; see, for example, [15, sections 3 and 4]. Therefore,

$$
\lim _{x_{k} \rightarrow \hat{x}} \frac{\xi_{2, F}\left(x_{k}, \alpha, \beta\right)}{\psi_{2, F}\left(x_{k}, \alpha, \beta\right)} \geq 1 .
$$

Remark 5.1. From the above proof we can easily observe that if we impose the constraint $\mathcal{R}(A+E)=\mathcal{R}(A)$ on $E$ in $(2.5)$, then $\xi_{2, F}\left(x_{k}, \alpha, \beta\right)=\psi_{2, F}\left(x_{k}, \alpha, \beta\right)$. This constraint may make sense in some situations.

Stewart [15, section 5] proved a result similar to but weaker than Theorem 5.1. Using $\hat{r} \equiv b-A \hat{x}$ to denote the true LS residual, he observed that the perturbations $E_{1}=\left(r_{k}-\hat{r}\right) x_{k}^{\dagger}$ and $f_{1}=0$ satisfy the normal equations constraint in (2.5), so that

$$
\xi_{2, F}\left(x_{k}, \alpha, \beta\right) \leq \min _{\xi}\left\{\xi:\left\|E_{1}\right\|_{2, F} \leq \xi \alpha\|A\|_{2, F}\right\}=\frac{\left\|E_{1}\right\|_{2, F}}{\alpha\|A\|_{2, F}}=\frac{\left\|r_{k}-\hat{r}\right\|}{\alpha\|A\|_{2, F}\left\|x_{k}\right\|}
$$

Since $r_{k}=P_{A} r_{k}+P_{A}^{\perp} r_{k}=P_{A} r_{k}+\hat{r}$, the bound in (5.6) is equivalent to

$$
\xi_{2, F}\left(x_{k}, \alpha, \beta\right) \leq \frac{\left\|P_{A} r_{k}\right\|}{\alpha\|A\|_{2, F}\left\|x_{k}\right\|} .
$$

By also considering perturbations in $b$, we obtain the new and tighter upper bound $\psi_{2, F}\left(x_{k}, \alpha, \beta\right)$ in (5.1), which is asymptotically tight with $\xi_{2, F}\left(x_{k}, \alpha, \beta\right)$ for all values of $\alpha$ and $\beta$.

Stewart noted in [16, section 3] that his bound in (5.6) could be computed only if the LS problem were contrived so that $\hat{r}$ was known a priori. Since this is generally not the case in practical applications, the bound in (5.6) cannot generally be used in practice. Of course, in practical applications $\left\|P_{A} r_{k}\right\|$ is also not available a priori and is too expensive to compute directly. However, by thinking of our new bound $\psi_{2, F}\left(x_{k}, \alpha, \beta\right)$ in (5.1) in terms of the projection $\left\|P_{A} r_{k}\right\|$ instead of the quantity $\| r_{k}-$ $\hat{r} \|$, we can try to find new ways to estimate $\xi_{2, F}\left(x_{k}, \alpha, \beta\right)$ by estimating $\left\|P_{A} r_{k}\right\|$. The following bounds, for example, follow immediately from Lemma 4.1:

$$
\frac{\left\|A^{T} r_{k}\right\|}{\sigma_{1}} \leq\left\|P_{A} r_{k}\right\| \leq \frac{\left\|A^{T} r_{k}\right\|}{\sigma_{n}}
$$

where $\sigma_{1}$ and $\sigma_{n}$ are the largest and smallest singular values of $A$, respectively. Note that $\left\|A^{T} r_{k}\right\|$ is generally easily computable and estimates of the extreme singular values of $A$ are available in LSQR.

For very well-conditioned problems, corresponding to $\sigma_{1} \approx \sigma_{n}$, the bounds in (5.7) are fairly tight. In our numerical tests we found that even for ill-conditioned problems the lower bound in (5.7) is usually much tighter than the upper bound. In other words, $\bar{\sigma}_{k}$ in Lemma 4.1 usually lies close to the largest singular value of $A$, especially late in phase 2 and in phase 3 of the iteration process. A better understanding of the behavior of $\bar{\sigma}_{k}$ with $k$ could lead to an efficiently computable estimate of $\left\|P_{A} r_{k}\right\|$ in (5.4). We leave this for a future investigation.

Here we suggest another potential approach for estimating the projection $\left\|P_{A} r_{k}\right\|$ efficiently in LSQR. Using the fact that $P_{A}=A A^{\dagger}$ and $P_{A} A=A$, and letting $\hat{x}=A^{\dagger} b$ denote the true LS solution of (1.1), we obtain

$$
\left\|P_{A} r_{k}\right\|=\left\|A A^{\dagger}\left(b-A x_{k}\right)\right\|=\left\|A\left(\hat{x}-x_{k}\right)\right\| \equiv\left\|\hat{x}-x_{k}\right\|_{A^{T} A} .
$$


The quantity $\left\|P_{A} r_{k}\right\|$ is therefore the so-called energy norm of the error at step $k$. Several estimates of the energy norm of the error have been proposed for the method of conjugate gradients; see, for example, the discussion in [17] and an extension to CGLS in [1]. We expect that it will be possible to use these ideas to estimate $\left\|P_{A} r_{k}\right\|$ efficiently in LSQR. We leave the details for future work.

6. A necessary and sufficient condition for $\xi_{2, F}\left(x_{k}, \alpha, \beta\right) \leq 1$. We now show how the groundbreaking theoretical results of Waldén, Karlson, and Sun, namely, [18, Theorem 2.2 and Corollary 2.1] can be used to give a necessary and sufficient condition to determine whether an iterate $x_{k}$ is an acceptable LS solution.

The minimization problems in Lemma 6.1 are commonly called normwise backward error or minimal backward error problems; see [9, section 7] and [18].

Lemma 6.1. Given $A \in \mathbb{R}^{m \times n}, b \in \mathbb{R}^{m}, 0 \neq x_{k} \in \mathbb{R}^{n}$, and $\theta>0$, define $r_{k} \equiv b-A x_{k}$. Then for compatible systems we have (see [9, Exercise 7.8, p. 134])

$$
\begin{aligned}
& \omega\left(x_{k}, \theta\right) \equiv \min _{\Delta A, \Delta b}\left\{\|[\Delta A, \theta \Delta b]\|_{F}:(A+\Delta A) x_{k}=b+\Delta b\right\}=\frac{\theta\left\|r_{k}\right\|}{\sqrt{1+\theta^{2}\left\|x_{k}\right\|^{2}}}, \\
& \omega\left(x_{k}, \infty\right) \equiv \lim _{\theta \rightarrow \infty} \omega\left(x_{k}, \theta\right)=\min _{\Delta A}\left\{\|\Delta A\|_{F}:(A+\Delta A) x_{k}=b\right\}=\frac{\left\|r_{k}\right\|}{\left\|x_{k}\right\|} .
\end{aligned}
$$

If we replace the above equality constraints by the LS normal equations, then with the above-defined $\omega\left(x_{k}, \theta\right)$ and $\omega\left(x_{k}, \infty\right)$, we have (see [18]; see also [9, section 20.7])

$$
\begin{aligned}
& \mu\left(x_{k}, \theta\right) \\
& \quad \equiv \min _{\Delta A, \Delta b}\left\{\|[\Delta A, \theta \Delta b]\|_{F}:(A+\Delta A)^{T}\left[(b+\Delta b)-(A+\Delta A) x_{k}\right]=0\right\} \\
& \quad=\min \left\{\omega\left(x_{k}, \theta\right), \sigma_{\min }\left(\left[A, \omega\left(x_{k}, \theta\right) \cdot\left(I-r_{k} r_{k}^{\dagger}\right)\right]\right)\right\}, \\
& \mu\left(x_{k}, \infty\right) \equiv \lim _{\theta \rightarrow \infty} \mu\left(x_{k}, \theta\right) \\
& \quad=\min _{\Delta A}\left\{\|\Delta A\|_{F}:(A+\Delta A)^{T}\left[b-(A+\Delta A) x_{k}\right]=0\right\} \\
& \quad=\min \left\{\omega\left(x_{k}, \infty\right), \sigma_{\min }\left(\left[A, \omega\left(x_{k}, \infty\right) \cdot\left(I-r_{k} r_{k}^{\dagger}\right)\right]\right)\right\},
\end{aligned}
$$

where $\sigma_{\min }(\cdot)$ denotes the smallest singular value.

The question is how to use $\mu\left(x_{k}, \theta\right)$ or $\mu\left(x_{k}, \infty\right)$ to determine whether $x_{k}$ is an acceptable LS solution as defined in (2.4) and (2.5). Let $\widehat{\Delta A}$ be the optimal perturbation in (6.4). If $\mu\left(x_{k}, \infty\right) \equiv\|\widehat{\Delta A}\|_{F} \leq \alpha\|A\|_{F}$, then clearly $E=\widehat{\Delta A}$ and $f=0$ satisfy the constraints in $(2.3)$ in the Frobenius norm and give $\xi_{F}\left(x_{k}, \alpha, \beta\right) \leq 1$ in (2.4). On the other hand, $\mathrm{Gu}[8$, Theorem 3.1] showed that for any $E, f, \alpha$, and $\beta$ such that

$$
(A+E)^{T}\left[b+f-(A+E) x_{k}\right]=0, \quad\|E\|_{2, F} \leq \alpha\|A\|_{2, F}, \quad\|f\| \leq \beta\|b\|,
$$

so that $\xi_{F}\left(x_{k}, \alpha, \beta\right) \leq 1$ (see $\left.(2.5)\right)$, there exists a $\Delta A$ satisfying the constraint in (6.4) with

$$
\frac{\|\Delta A\|_{2, F}}{\|A\|_{2, F}} \leq \alpha+2 \beta \frac{1+\alpha}{1-2 \beta},
$$

ensuring that $\mu\left(x_{k}, \infty\right) \lesssim(\alpha+2 \beta)\|A\|_{F}$ in (6.4) when $\alpha, \beta \ll 1$. So for any $\alpha$ and $\beta$ satisfying $0 \leq \alpha, \beta \ll 1$, we have

$$
\begin{aligned}
& \mu\left(x_{k}, \infty\right) \leq \alpha\|A\|_{F} \Rightarrow \xi_{F}\left(x_{k}, \alpha, \beta\right) \leq 1 \\
& \xi_{F}\left(x_{k}, \alpha, \beta\right) \leq 1 \Rightarrow \mu\left(x_{k}, \infty\right) \lesssim(\alpha+2 \beta)\|A\|_{F} .
\end{aligned}
$$

Copyright (C) by SIAM. Unauthorized reproduction of this article is prohibited. 
From this we see that if $\beta \lesssim \alpha$, then checking the condition $\mu\left(x_{k}, \infty\right) \leq \alpha\|A\|_{F}$ is a reliable way to determine whether $x_{k}$ is an acceptable solution in the Frobenius norm, and in particular for checking whether it is a backward stable LS solution.

Unfortunately, when $\alpha \ll \beta$, the condition $\mu\left(x_{k}, \infty\right) \leq \alpha\|A\|_{F}$ is sufficient only for $x_{k}$ to be an acceptable solution. In other words, when $\alpha \ll \beta$,

$$
\xi_{F}\left(x_{k}, \alpha, \beta\right) \leq 1 \nRightarrow \mu\left(x_{k}, \infty\right) \lesssim \alpha\|A\|_{F},
$$

and in this case the criterion $\mu\left(x_{k}, \infty\right) \leq \alpha\|A\|_{F}$ might not detect that an acceptable iterate has been obtained until many unnecessary iterations have been performed, if it detects it at all. Recall from section 2 that the case $\alpha \ll \beta$ often occurs in practical LS applications, because $b$ is often a measurement vector that is subject to much larger measurement errors than the matrix $A$.

We now consider how to use $\mu\left(x_{k}, \theta\right)$ with an appropriate finite $\theta$ in (6.3) to determine whether $x_{k}$ is an acceptable LS solution for any choice of $\alpha$ and $\beta$ in (2.5). The following lemma for compatible systems was proven in [3, section 2].

LEMma 6.2. Using the notation of Lemma 6.1 , if $\beta\|b\|>0$, then the choice

$$
\theta=\hat{\theta}_{2, F} \equiv\left(\frac{\alpha\|A\|_{2, F}}{\beta\|b\| \cdot\left\|x_{k}\right\|}\right)^{1 / 2}
$$

makes the optimal $\Delta A$ and $\Delta b$ in (6.1) equal to the optimal $E$ and $f$ in (3.3).

Note that the relationship between $\omega\left(x_{k}, \theta\right)$ in $(6.1)$ and $\eta_{2, F}\left(x_{k}, \alpha, \beta\right)$ in (3.3) for compatible systems parallels that between $\mu\left(x_{k}, \theta\right)$ in (6.3) and $\xi_{2, F}\left(x_{k}, \alpha, \beta\right)$ in (2.5) for LS problems. We have not found an exact equivalence to Lemma 6.2 for the LS case, but we do have a very strong result, which we give in the following theorem.

TheOREM 6.3. Given full column rank $A \in \mathbb{R}^{m \times n}, b \in \mathbb{R}^{m}$, and $0 \neq x_{k} \in \mathbb{R}^{n}$, define $r_{k} \equiv b-A x_{k}, \xi_{F}\left(x_{k}, \alpha, \beta\right)$ as in (2.5) and $\mu\left(x_{k}, \theta\right)$ as in (6.3). Let

$$
\theta=\hat{\theta} \equiv \frac{\alpha\|A\|_{F}}{\beta\|b\|} .
$$

Then,

$$
\xi_{F}\left(x_{k}, \alpha, \beta\right) \leq \frac{\mu\left(x_{k}, \hat{\theta}\right)}{\alpha\|A\|_{F}} \leq \sqrt{2} \xi_{F}\left(x_{k}, \alpha, \beta\right)
$$

Proof. Let $\widehat{\Delta A}$ and $\widehat{\Delta b}$ represent the optimal perturbations in (6.3) with $\theta=\hat{\theta}$. Clearly $E=\widehat{\Delta A}$ and $f=\widehat{\Delta b}$ cannot improve on the optimal $\xi_{F}\left(x_{k}, \alpha, \beta\right)$ in $(2.5)$, and so either $\|\widehat{\Delta A}\|_{F} \geq \xi_{F}\left(x_{k}, \alpha, \beta\right) \alpha\|A\|_{F}$ or $\|\widehat{\Delta b}\| \geq \xi_{F}\left(x_{k}, \alpha, \beta\right) \beta\|b\|$, or both, giving

$$
\max \left\{\frac{\|\widehat{\Delta A}\|_{F}}{\alpha\|A\|_{F}}, \frac{\|\widehat{\Delta b}\|}{\beta\|b\|}\right\} \geq \xi_{F}\left(x_{k}, \alpha, \beta\right) .
$$

Thus we have

$$
\begin{aligned}
\mu\left(x_{k}, \hat{\theta}\right)=\|[\widehat{\Delta A}, \hat{\theta} \widehat{\Delta b}]\|_{F} & =\sqrt{\|\widehat{\Delta A}\|_{F}^{2}+\frac{\alpha^{2}\|A\|_{F}^{2}}{\beta^{2}\|\widehat{\Delta b}\|^{2}}} \\
& \geq \max \left\{\|\widehat{\Delta A}\|_{F}, \frac{\alpha\|A\|_{F}}{\beta\|\widehat{\Delta b}\|} \|\right\} \\
& \geq \xi_{F}\left(x_{k}, \alpha, \beta\right) \alpha\|A\|_{F},
\end{aligned}
$$

Copyright $@$ by SIAM. Unauthorized reproduction of this article is prohibited. 
proving the first inequality in (6.7).

On the other hand, for the optimal $\hat{E}$ and $\hat{f}$ in (2.5), we have

$$
\|\hat{E}\|_{F} \leq \xi_{F}\left(x_{k}, \alpha, \beta\right) \alpha\|A\|_{F}, \quad\|\hat{f}\| \leq \xi_{F}\left(x_{k}, \alpha, \beta\right) \beta\|b\|,
$$

where $\hat{E}$ and $\hat{f}$ cannot improve on the optimal $\widehat{\Delta A}$ and $\widehat{\Delta b}$ in (6.3) with $\theta=\hat{\theta}$. Therefore,

$$
\begin{aligned}
\mu\left(x_{k}, \hat{\theta}\right) & =\|[\widehat{\Delta A}, \hat{\theta} \widehat{\Delta b}]\|_{F} \leq\|[\hat{E}, \hat{\theta} \hat{f}]\|_{F} \\
& \leq \sqrt{\xi_{F}^{2}\left(x_{k}, \alpha, \beta\right) \alpha^{2}\|A\|_{F}^{2}+\frac{\alpha^{2}\|A\|_{F}^{2}}{\beta^{2}\|b\|^{2}} \xi_{F}^{2}\left(x_{k}, \alpha, \beta\right) \beta^{2}\|b\|^{2}} \\
& =\sqrt{2} \xi_{F}\left(x_{k}, \alpha, \beta\right) \alpha\|A\|_{F},
\end{aligned}
$$

leading to the second inequality in (6.7).

Theorem 6.3 dealt with $\xi_{F}\left(x_{k}, \alpha, \beta\right)$ and $\mu\left(x_{k}, \hat{\theta}\right)$ defined for the perturbed LS normal equations $(A+E)^{T}\left[b+f-(A+E) x_{k}\right]=0$. But the same analysis can be applied to $\eta_{F}\left(x_{k}, \alpha, \beta\right)$ in $(3.3)$ and $\omega\left(x_{k}, \hat{\theta}\right)$ in $(6.1)$, which were defined for the perturbed compatible system $(A+E) x_{k}=b+f$. Carrying out the argument in Theorem 6.3 with $\xi_{F}\left(x_{k}, \alpha, \beta\right)$ replaced by $\eta_{F}\left(x_{k}, \alpha, \beta\right)$, and $\mu\left(x_{k}, \hat{\theta}\right)$ replaced by $\omega\left(x_{k}, \hat{\theta}\right)$, gives

$$
\eta_{F}\left(x_{k}, \alpha, \beta\right) \leq \frac{\omega\left(x_{k}, \hat{\theta}\right)}{\alpha\|A\|_{F}} \leq \sqrt{2} \eta_{F}\left(x_{k}, \alpha, \beta\right) .
$$

Recall from section 2 that $x_{k}$ is an acceptable LS solution if and only if it satisfies $\xi_{2, F}\left(x_{k}, \alpha, \beta\right) \leq 1$, but that at present no explicit formula is known for computing $\xi_{2, F}\left(x_{k}, \alpha, \beta\right)$. Theorem 6.3 implies that

$$
\begin{aligned}
& \mu\left(x_{k}, \hat{\theta}\right) \leq \alpha\|A\|_{F} \Rightarrow \xi_{F}\left(x_{k}, \alpha, \beta\right) \leq 1, \\
& \xi_{F}\left(x_{k}, \alpha, \beta\right) \leq 1 \Rightarrow \mu\left(x_{k}, \hat{\theta}\right) \leq \sqrt{2} \alpha\|A\|_{F} .
\end{aligned}
$$

(Compare this to (6.6) in which $\beta$ is present in the second expression-a subtle but very important difference when $\alpha \ll \beta$.) The following is therefore a nearly optimal test for an acceptable Frobenius norm LS solution for any choice of $\alpha$ and $\beta$ :

$$
\text { LStest2: } \quad \mu\left(x_{k}, \hat{\theta}\right) \leq \alpha\|A\|_{F}, \quad \text { where } \hat{\theta}=\frac{\alpha\|A\|_{F}}{\beta\|b\|} .
$$

As a consequence of Theorem 6.3 we can now determine almost exactly when an iterate $x_{k}$ is an acceptable LS solution by using the result of Waldén, Karlson, and Sun [18] in Lemma 6.1. Unfortunately, Lemma 6.1 gives an expression for $\mu\left(x_{k}, \theta\right)$ that costs $\mathcal{O}\left(\mathrm{m}^{3}\right)$ flops to compute, and is thus too expensive to be useful in large sparse applications. Nevertheless we can still use (6.9) to test the effectiveness of LSQR's stopping criteria 1 and 2 as well as our new condition LStest1 (5.3). We can also try to develop more effective stopping criteria for the iterative solution of large sparse LS problems by finding bounds on or estimates for $\mu\left(x_{k}, \hat{\theta}\right)$ instead of $\xi_{2, F}\left(x_{k}, \alpha, \beta\right)$.

A few estimates of $\mu\left(x_{k}, \infty\right)$ exist in the literature; see, for example, $[7$, section 4] and the references therein. We point out that some of these can be generalized to estimate $\mu\left(x_{k}, \hat{\theta}\right)$, but to our knowledge none of these estimates is both provably reliable and computable in $\mathcal{O}(m n)$ flops, so at present no estimate of $\mu\left(x_{k}, \hat{\theta}\right)$ is truly suitable for use in large sparse applications. We leave the efficient and reliable estimation of $\mu\left(x_{k}, \hat{\theta}\right)$ for a future investigation. 
7. Illustrations. To illustrate our results, we run LSQR on various test problems and plot at each iteration the following:

(i) the residual norm $\left\|r_{k}\right\|$,

(ii) Stewart's $\left\|A^{T} r_{k}\right\| /\left\|r_{k}\right\|$,

(iii) the norm of the projections $\left\|P_{A} r_{k}\right\|$ and $\left\|P_{A}^{\perp} r_{k}\right\|$,

(iv) the ratio $\bar{\sigma}_{k}=\left\|A^{T} r_{k}\right\| /\left\|P_{A} r_{k}\right\|$ from (4.3).

The projections are computed by obtaining the QR factorization of $A$ using MATLAB's built-in function qr, and the quantities $\left\|r_{k}\right\|,\left\|A^{T} r_{k}\right\|,\|A\|_{2}$, and $\|A\|_{F}$ are computed explicitly. (In other words, we do not use LSQR's approximation to the above quantities, because our goal here is to illustrate the actual convergence behavior, described in section 4.1, of the true $\left\|r_{k}\right\|$ and $\left\|A^{T} r_{k}\right\| /\left\|r_{k}\right\|$.) We also show at which iteration phases 1 and 2 end with vertical lines, indicating the first $k$ for which (4.7) and (4.8) hold, respectively. Admittedly, the $\mathcal{O}(u)$ term in (4.8) is somewhat vague; for our illustrations we plot the vertical line at the first $k$ for which $\left\|P_{A} r_{k}\right\|$ settles near $u\left(\|A\|_{2}\left\|x_{k}\right\|+\|b\|\right)$. Also note that only one vertical line is plotted in test problems 1 and 4 because there is effectively no phase 2 or 1 , respectively, in these problems.

We use test problems 1 to 4 to illustrate how the effectiveness of LSQR's stopping criteria 1 and 2 in (3.2) depends on the size of the true residual norm $\|\hat{r}\|$. For these simple test problems, each element of the matrix $A \in \mathbb{R}^{300 \times 120}$ is randomly chosen from a normal distribution with mean 0 and standard deviation 1 , so that $A$ is almost certainly well-conditioned. This exhibits the interesting observation discussed in section 4.1, and supports our explanation beautifully. Let $s_{n}$ represent an $n$-vector containing all ones, and let each element of an $m$-vector $t_{m}$ be randomly chosen from a normal distribution with mean 0 and standard deviation 1 .

(i) In test problem $1, b=A s_{120}+10^{-15} t_{300}$ and $\|\hat{r}\| \approx 10^{-14}$.

(ii) In test problem $2, b=A s_{120}+10^{-10} t_{300}$ and $\|\hat{r}\| \approx 10^{-9}$.

(iii) In test problem $3, b=A s_{120}+10^{-5} t_{300}$ and $\|\hat{r}\| \approx 10^{-4}$.

(iv) In test problem $4, b=A s_{120}+10^{0} t_{300}$ and $\|\hat{r}\| \approx 10^{1}$.

Results for these test problems are illustrated in Figure 3.

We use test problems 5 to 8 to illustrate how increasing the condition number $\kappa_{2}(A)$ affects the behavior of $\left\|r_{k}\right\|$ and $\left\|A^{T} r_{k}\right\| /\left\|r_{k}\right\|$ in (3.2) and $\bar{\sigma}_{k}$ in (4.3). These problems are set up so that they all have roughly the same true LS residual $\|\hat{r}\| \approx 10^{-7}$, and are presented by increasing condition number, with $\kappa_{2}(A) \approx 7.1,4.5 \times 10^{3}$, $1.5 \times 10^{8}$, and $3.8 \times 10^{12}$, respectively. Although the focus of this paper is not on regularization of ill-conditioned problems, we include these examples to illustrate the numerical behavior of LSQR, and our observation from section 4.1, on these increasingly ill-conditioned problems.

For the test problems 5 to 8 we create $A$ and $b$ as in [12, section 8]. These problems are called $P(m, n, d, p)$. The matrix $A \in \mathbb{R}^{m \times n}$ has singular values

$$
\sigma_{i} \equiv(\lfloor(i-1+d) / d\rfloor \cdot d / n)^{p}
$$

where integer division by $d$ is used to obtain repeated singular values. The true solution $\hat{x}$ and residual $\hat{r}$ are fixed, after which $b$ is set to $b=A \hat{x}+\hat{r}$. For all the details see [12, p. 63]. Test problems 5 to 8 are $P(500,200,4,1 / 2), P(800,200,3,2)$, $P(750,300,7,5)$, and $P(400,150,6,9)$, respectively. Results for these problems are illustrated in Figure 4.

For test problems 9 to 12 we use the large sparse sample problems Well1033, Well1850, Illc1033, and Illc1850 from the Matrix Market [10], respectively. The 

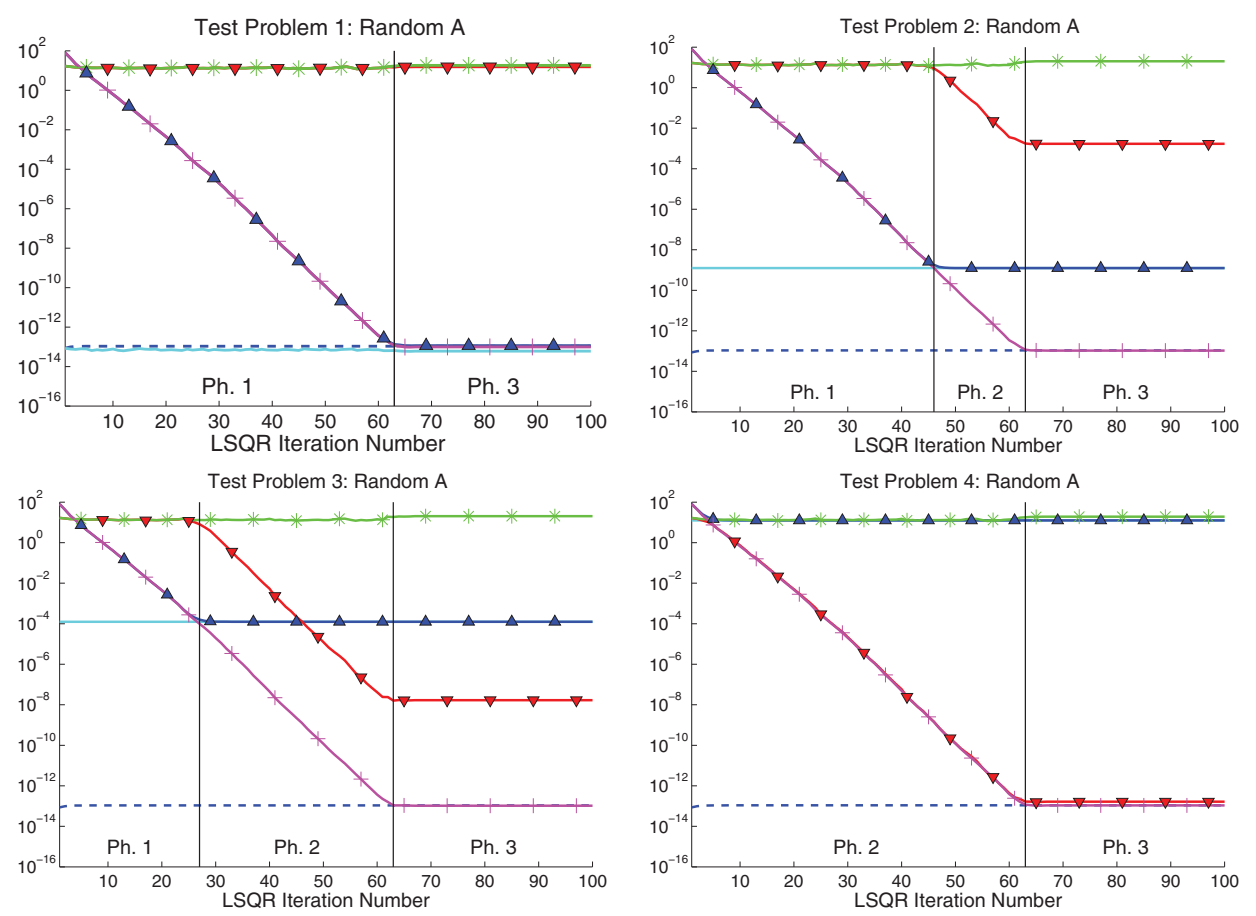

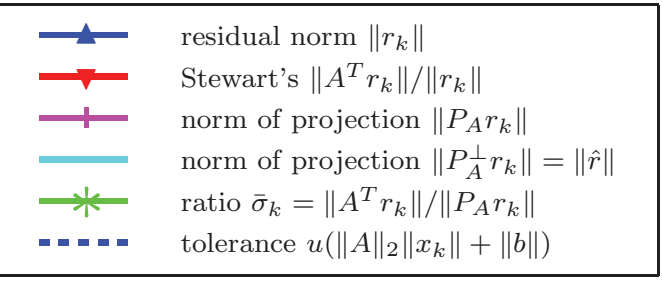

FIG. 3. Test problems 1 to 4: well-conditioned examples with increasing true $\|\hat{r}\|$.

problems starting with "Well" denote well-conditioned problems, whereas those starting with "Illc" denote ill-conditioned problems. The problems ending with the number 1033 involve a matrix $A \in \mathbb{R}^{1033 \times 320}$, whereas those ending with 1850 involve a matrix $A \in \mathbb{R}^{1850 \times 712}$. In all these test problems we create the vector $b$ as follows: $b=A[1,1, \ldots, 1]^{T}+10^{-8}[m, m-1, \ldots, 1]^{T}$. Results for these problems are illustrated in Figure 5.

Each convergence plot in Figures 3 to 5 corresponds to one instance of a LS problem. Tables 1 and 2 below give the number of iterations required to trigger the following, for various values of $\alpha$ and $\beta$ :

(i) LSQR's stopping criteria 1 and 2 from (3.2), in the Frobenius norm,

(ii) the new condition LStest1 from (5.3),

(iii) the new condition LStest2 from (6.9).

The iteration counts in (i) above are given when the relevant norms in (3.2) are computed explicitly (True) and using LSQR's approximation (App.). Each row corresponds to an average number of iterations required, rounded to the nearest integer, for the same matrix $A$ with 100 different noisy vectors $b=A s_{n}+10^{-p} t_{m}$ (where $s_{n}$ 

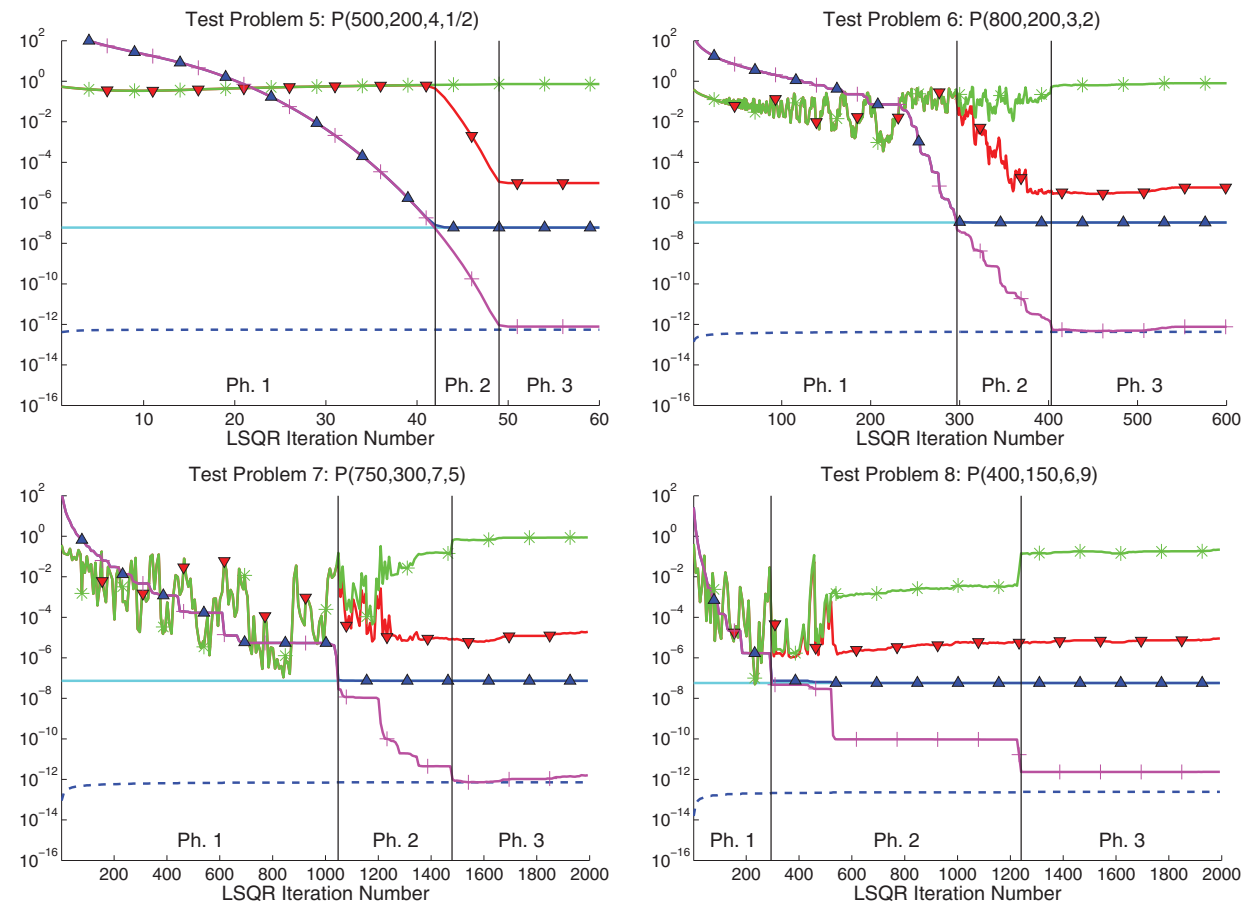

\begin{tabular}{|c|c|}
\hline$\underset{+\infty}{+\infty}$ & $\begin{array}{l}\text { residual norm }\left\|r_{k}\right\| \\
\text { Stewart's }\left\|A^{T} r_{k}\right\| /\left\|r_{k}\right\| \\
\text { norm of projection }\left\|P_{A} r_{k}\right\| \\
\text { norm of projection }\left\|P_{A}^{\perp} r_{k}\right\|=\|\hat{r}\| \\
\text { ratio } \bar{\sigma}_{k}=\left\|A^{T} r_{k}\right\| /\left\|P_{A} r_{k}\right\| \\
\text { tolerance } u\left(\|A\|_{2}\left\|x_{k}\right\|+\|b\|\right)\end{array}$ \\
\hline
\end{tabular}

FiG. 4. Test problems 5 to 8: increasingly ill-conditioned examples with $\|\hat{r}\| \approx 10^{-7}$.

and $t_{m}$ are defined above). The symbol $\infty$ is used when a particular condition is never satisfied in any of the 100 tests, regardless of the number of LSQR iterations performed. We compute the quantity $\mu\left(x_{k}, \hat{\theta}\right)$ in $(6.3)$, with $\hat{\theta}$ in Theorem 6.3 , using MATLAB's built-in command svd.

We use test problems 1 to 4 in Table 1 (with $p=15,10,5$, and 0 , respectively) to demonstrate the impact of the size of the true residual norm on the effectiveness of the stopping criteria. We use test problems 9 and 11 in Table 2 (with $p=7$ ) to test LSQR's stopping criteria on matrices from the Matrix Market. Ideally, methods such as LSQR are applied to systems which (possibly after preconditioning) are well-conditioned, but this is not always possible, and an understanding of the behavior of these methods on ill-conditioned problems can be helpful in some practical cases, as well as giving us greater insight into the general numerical behavior of these algorithms. For this reason, we give numerical results for both a well-conditioned and an ill-conditioned test problem in Table 2.

8. Discussion and conclusions. As a general trend, in the well-conditioned test problems 1 to 5 , the ratio $\bar{\sigma}_{k}=\left\|A^{T} r_{k}\right\| /\left\|P_{A} r_{k}\right\|$ remains relatively constant. As 

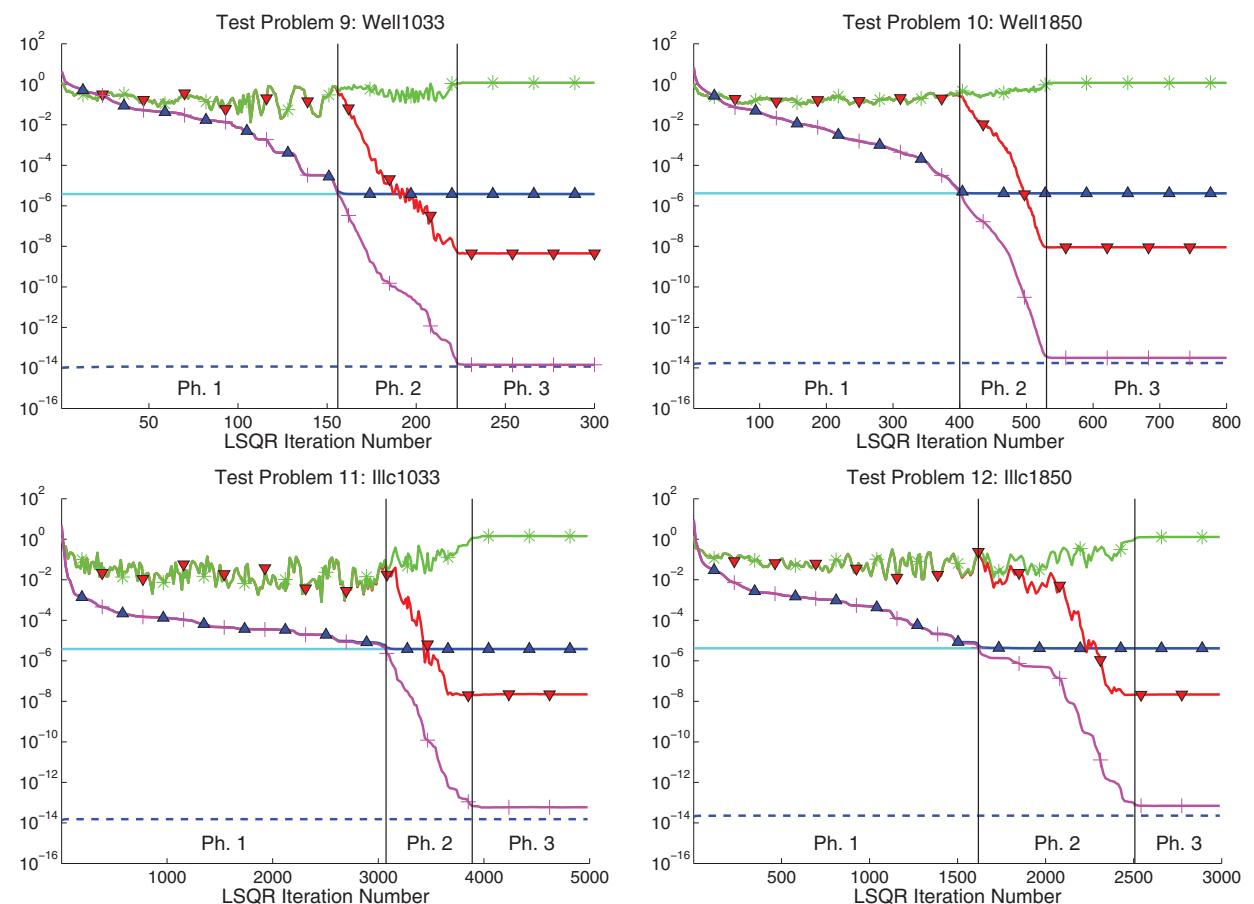

\begin{tabular}{|c|c|}
\hline- & residual norm $\left\|r_{k}\right\|$ \\
\hline$\rightarrow$ & Stewart's $\left\|A^{T} r_{k}\right\| /\left\|r_{k}\right\|$ \\
\hline - & norm of projection $\left\|P_{A} r_{k}\right\|$ \\
\hline * & $\begin{array}{l}\text { norm of projection }\left\|P_{A}^{\perp} r_{k}\right\|=\|\hat{r}\| \\
\text { ratio } \bar{\sigma}_{k}=\left\|A^{T} r_{k}\right\| /\left\|P_{A} r_{k}\right\|\end{array}$ \\
\hline--2 & tolerance $u\left(\|A\|_{2}\left\|x_{k}\right\|+\|b\|\right)$ \\
\hline
\end{tabular}

FiG. 5. Test problems 9 to 12 : sparse examples from the Matrix Market with $\|\hat{r}\| \approx 10^{-6}$.

the problems become more and more ill-conditioned, here in test problems 6 to $8, \bar{\sigma}_{k}$ has a much more oscillatory behavior, as expected.

In most of the test problems, there is a very clear visual distinction in the convergence plots between phases 1 and 2 , and also between phases 2 and 3 . In phase 1 we clearly observe that $\left\|r_{k}\right\| \approx\left\|P_{A} r_{k}\right\|$ decreases while Stewart's $\left\|A^{T} r_{k}\right\| /\left\|r_{k}\right\| \approx \bar{\sigma}_{k}$ oscillates between the extreme singular values of $A$ (which means it remains roughly constant for the well-conditioned problems and can vary wildly for the ill-conditioned problems). In phase 2 on the other hand, we see that $\left\|r_{k}\right\| \approx\left\|P_{A}^{\perp} r_{k}\right\|$ remains nearly constant while $\left\|A^{T} r_{k}\right\| /\left\|r_{k}\right\|$ starts to decrease. Note that in phase $2\left\|A^{T} r_{k}\right\| /\left\|r_{k}\right\|$ decreases at almost exactly the same rate as $\left\|P_{A} r_{k}\right\|$, as suggested by Lemma 4.1 and the fact that $\left\|r_{k}\right\| \approx\|\hat{r}\|$ is nearly constant in phase 2 . Also note that there is no phase 2 in test problem 1 because $\left\|r_{k}\right\|$ satisfies (4.9) when $k \approx 63$, and no phase 1 in test problem 4 because $\left\|r_{0}\right\| \approx\|\hat{r}\|$; see the comments after (4.6). Finally, in phase 3 , both $\left\|r_{k}\right\|$ and $\left\|A^{T} r_{k}\right\| /\left\|r_{k}\right\|$ remain nearly constant.

The above patterns are most obvious in the well-conditioned problems and less so in the very ill-conditioned problems. For example, in test problems 7 and $8, \bar{\sigma}_{k}$ 
TABLE 1

The effectiveness of LSQR's stopping criteria depends on the size of $\|\hat{r}\|$.

\begin{tabular}{|c|cc|cc|cc|c|c|}
\hline Test & \multicolumn{2}{|c|}{ Parameters } & \multicolumn{2}{c|}{ LSQR 1 } & \multicolumn{2}{c|}{ LSQR 2 } & LStest1 & LStest2 \\
problem & $\alpha$ & $\beta$ & True & App. & True & App. & $(5.3)$ & $(6.9)$ \\
\hline \multirow{5}{*}{1} & $10^{-4}$ & $10^{-4}$ & 13 & 13 & $\infty$ & 73 & 13 & 13 \\
$\|\hat{r}\| \approx 10^{-14}$ & $10^{-8}$ & $10^{-4}$ & 18 & 18 & $\infty$ & 85 & 18 & 18 \\
& $10^{-8}$ & $10^{-8}$ & 30 & 30 & $\infty$ & 85 & 30 & 30 \\
& $10^{-12}$ & $10^{-8}$ & 34 & 34 & $\infty$ & 98 & 34 & 34 \\
& $10^{-14}$ & $10^{-14}$ & 53 & 53 & $\infty$ & 103 & 53 & 53 \\
\hline \multirow{3}{*}{2} & $10^{-4}$ & $10^{-4}$ & 13 & 13 & 58 & 58 & 13 & 13 \\
$\|\hat{r}\| \approx 10^{-9}$ & $10^{-8}$ & $10^{-4}$ & 18 & 18 & $\infty$ & 72 & 18 & 18 \\
& $10^{-8}$ & $10^{-8}$ & 30 & 30 & $\infty$ & 72 & 30 & 30 \\
& $10^{-12}$ & $10^{-8}$ & 34 & 34 & $\infty$ & 84 & 34 & 34 \\
& $10^{-14}$ & $10^{-14}$ & $\infty$ & $\infty$ & $\infty$ & 91 & 53 & 53 \\
\hline \multirow{5}{*}{$3 \hat{r} \| \approx 10^{-4}$} & $10^{-4}$ & $10^{-4}$ & 13 & 13 & 38 & 38 & 13 & 13 \\
& $10^{-8}$ & $10^{-4}$ & 18 & 18 & 54 & 54 & 18 & 18 \\
& $10^{-8}$ & $10^{-8}$ & $\infty$ & $\infty$ & 54 & 54 & 30 & 30 \\
& $10^{-12}$ & $10^{-8}$ & $\infty$ & $\infty$ & $\infty$ & 69 & 34 & 34 \\
& $10^{-14}$ & $10^{-14}$ & $\infty$ & $\infty$ & $\infty$ & 75 & 53 & 53 \\
\hline \multirow{4}{*}{$4 \hat{r} \| \approx 10^{1}$} & $10^{-4}$ & $10^{-4}$ & $\infty$ & $\infty$ & 13 & 13 & 14 & 13 \\
& $10^{-8}$ & $10^{-4}$ & $\infty$ & $\infty$ & 30 & 30 & 18 & 18 \\
& $10^{-8}$ & $10^{-8}$ & $\infty$ & $\infty$ & 30 & 30 & 31 & 30 \\
& $10^{-12}$ & $10^{-8}$ & $\infty$ & $\infty$ & 46 & 46 & 35 & 35 \\
& $10^{-14}$ & $10^{-14}$ & $\infty$ & $\infty$ & 54 & 54 & 54 & 53 \\
\hline
\end{tabular}

TABLE 2

Testing LSQR's stopping criteria on problems from the Matrix Market.

\begin{tabular}{|c|cc|cc|cc|c|c|}
\hline Test & \multicolumn{2}{|c|}{ Parameters } & \multicolumn{2}{|c|}{ LSQR 1 } & \multicolumn{2}{c|}{ LSQR 2 } & LStest1 & LStest2 \\
problem & $\alpha$ & $\beta$ & True & App. & True & App. & $(5.3)$ & $(6.9)$ \\
\hline \multirow{3}{*}{9} & $10^{-4}$ & $10^{-4}$ & 69 & 69 & 172 & 172 & 69 & 69 \\
Well1033 & $10^{-8}$ & $10^{-4}$ & 111 & 111 & 207 & 207 & 111 & 111 \\
$\|\hat{r}\| \approx 10^{-6}$ & $10^{-8}$ & $10^{-8}$ & 159 & 159 & 207 & 207 & 158 & 158 \\
& $10^{-12}$ & $10^{-8}$ & $\infty$ & $\infty$ & $\infty$ & 242 & 164 & 164 \\
& $10^{-14}$ & $10^{-14}$ & $\infty$ & $\infty$ & $\infty$ & 267 & 206 & 206 \\
\hline \multirow{3}{*}{11} & $10^{-4}$ & $10^{-4}$ & 43 & 43 & 1346 & 1346 & 43 & 43 \\
Illc1033 & $10^{-8}$ & $10^{-4}$ & 110 & 110 & 3563 & 3562 & 110 & 110 \\
$\|\hat{r}\| \approx 10^{-6}$ & $10^{-8}$ & $10^{-8}$ & 3066 & 3066 & 3563 & 3562 & 3045 & 3049 \\
& $10^{-12}$ & $10^{-8}$ & $\infty$ & $\infty$ & $\infty$ & 4049 & 3154 & 3154 \\
& $10^{-14}$ & $10^{-14}$ & $\infty$ & $\infty$ & $\infty$ & 4610 & 3610 & 3614 \\
\hline
\end{tabular}

oscillates a great deal and $\left\|P_{A} r_{k}\right\|$ decreases very slowly and in a staircase pattern, making the boundaries between successive phases less clear.

We note that the amplitude of the phase 1 oscillations in $\bar{\sigma}_{k}$ (and from (4.6) also in $\left.\left\|A^{T} r_{k}\right\| /\left\|r_{k}\right\|\right)$ are sometimes very large when LSQR is near stalling; see, for example, the plots for test problems 6 to 9. It would be interesting (but probably very difficult) to try to understand this phenomenon more clearly.

It is important to note that in every plot, $\left\|P_{A} r_{k}\right\|$ decreases monotonically to a level determined by the machine precision (see the comments following (4.2), (4.8), and Theorem 5.1), after which it remains nearly constant. This is a property of the minimum residual method, here LSQR. One contribution of this paper is to show how this decrease is first exhibited in the decrease of $\left\|r_{k}\right\|$ until it reaches its computational plateau, and is then exhibited in the decrease of the previously fairly level on average, but sometimes quite oscillatory, $\left\|A^{T} r_{k}\right\| /\left\|r_{k}\right\|$. Since $\left\|P_{A} r_{k}\right\|$ itself is not in practice directly available (see the comments in section 5 ), this might in itself be 
useful information. Note from (4.2) and Lemma 4.1 that this is essentially a property of $\|\hat{r}\|$ and the decreasing $\left\|r_{k}\right\|$, not a property reserved to LSQR.

We note that these examples and this analysis shed light on the convergence of all well-behaved minimum residual iterative methods for the LS problem. In particular they give a good understanding of why Stewart's $\left\|A^{T} r_{k}\right\| /\left\|r_{k}\right\|$ usually shows no significant improvement at all during what we have described as phase 1 . In fact, only when $\left\|r_{k}\right\|$ has effectively reached its plateau does Stewart's $\left\|A^{T} r_{k}\right\| /\left\|r_{k}\right\|$ start to decrease - which it tends to do throughout phase 2 - until it too finally reaches a plateau in phase 3 . We have thus provided an explanation for this previously puzzling result for minimum residual LS iterations. Thinking of the convergence of LSQR in terms of projections of the residuals has also led to the potentially very useful Theorems 5.1 and 5.2 .

Theorem 6.3 allows us to determine almost exactly at which iteration $x_{k}$ is an acceptable solution - although this comes at a very high computational cost. As mentioned in section 7, Tables 1 and 2 give the number of iterations required to trigger the various stopping criteria. By examining these two tables to find at which iteration the condition LStest2 is first satisfied, we see that LSQR's stopping criteria 1 and 2 in (3.2) can be much too pessimistic. In the nearly compatible test problem 1, LSQR's stopping criterion 1 is triggered exactly when an acceptable solution is obtained. This is not surprising considering that this criterion is ideal for compatible systems; see (3.3) and (3.5). On the other hand, in the very large-residual test problem 4, it is criterion 2 that is more reliable because there is effectively no phase 1 , and $\left\|A^{T} r_{k}\right\| /\left\|r_{k}\right\|$ starts decreasing from the first few iterations. It is still triggered a little late when we set $\alpha \ll \beta$ (which is usually reasonable in practical applications, as discussed in section 6). This is to be expected because the quantity $\left\|A^{T} r_{k}\right\| /\left\|r_{k}\right\|$ is a backward error in $A$ only (see the comments after (3.7)) and thus LSQR's stopping criterion 2 does not use $\beta$ in any way.

Away from these two extremes, however, both of LSQR's stopping criteria (with norms computed explicitly) can be much too pessimistic. In fact, in all problems we have tested in which

$$
u\left(\|A\|_{F}\|\hat{x}\|+\|b\|\right) \ll\|\hat{r}\| \ll\left\|r_{0}\right\|,
$$

neither the residual norm $\left\|r_{k}\right\|_{2}$ nor Stewart's $\left\|A^{T} r_{k}\right\| /\left\|r_{k}\right\|$ reaches its respective tolerances in (3.2) when $\alpha$ and $\beta$ are chosen sufficiently small. (For example, in all test problems except problems 1 and 4 , in Tables 1 and 2 there are cases where both "True" LSQR stopping criteria fail to detect a backward stable iterate.) In contrast, our two new conditions LStest1 (5.3) and LStest2 (6.9) detect acceptable iterates for all choices of $\alpha$ and $\beta$ satisfying $\alpha, \beta \geq \mathcal{O}(u)$.

We note that stopping criterion 2 in (3.2) is usually triggered if LSQR's approximation to $\left\|A^{T} r_{k}\right\| /\left\|r_{k}\right\|$ is used to test the criterion, because this approximation does not usually plateau at the end of phase 2 (while $\left\|A^{T} r_{k}\right\| /\left\|r_{k}\right\|$ actually does); see Figure 2. In this case convergence is reported, even though in fact the actual $\left\|A^{T} r_{k}\right\| /\left\|r_{k}\right\| \gg \alpha\|A\|_{2, F}$. Criterion 2 using LSQR's approximation to $\left\|A^{T} r_{k}\right\| /\left\|r_{k}\right\|$ is usually triggered in practice in what we have called phase 3 , some iterations after LSQR has actually converged to a backward stable LS iterate.

Each iteration count in Tables 1 and 2 corresponds to an average using 100 different noisy vectors $b$. The iteration count for each individual test was almost always within $\approx 5 \%$ of the average. In almost all of our numerical tests, our new conditions LStest1 and LStest2 are triggered at almost exactly the same iteration. This seems 
to indicate that the asymptotically optimal criterion LStest $1\left(\psi_{F}\left(x_{k}, \alpha, \beta\right) \leq 1\right.$ from (5.3)) is just as reliable as the criterion LStest $2\left(\mu\left(x_{k}, \hat{\theta}\right) \leq \alpha\|A\|_{F}\right.$ from (6.9)), which we proved necessary and sufficient to within a factor of $\sqrt{2}$ in section 6 .

Finally, we note that although the above new conditions are very reliable for detecting when an acceptable LS solution has been obtained, neither can at present be estimated both reliably and efficiently enough to be used in practical large sparse applications. In the future we intend to examine whether reliable estimates of $\left\|P_{A} r_{k}\right\|$ and $\mu\left(x_{k}, \hat{\theta}\right)$ can be computed efficiently. We are optimistic that this is the case for $\left\|P_{A} r_{k}\right\|$, as noted in the last three paragraphs of section 5 . Such estimates could be used in LStest1 and LStest2 and would make ideal stopping criteria for the iterative solution of large sparse LS problems.

Acknowledgments. The authors are very grateful to Sou-Cheng Choi, Michael Saunders, and two anonymous referees. All their detailed and very helpful comments and suggestions have greatly improved the paper.

\section{REFERENCES}

[1] M. Arioli and S. Gratton, Least-Squares Problems, Normal Equations, and Stopping Criteria for the Conjugate Gradient Method, Technical report RAL-TR-2008-008, Rutherford Appleton Laboratory, Oxfordshire, UK, 2008.

[2] Å. BJörcK, Numerical Methods for Least Squares Problems, SIAM, Philadelphia, 1996.

[3] X.-W. Chang, C. C. Paige, and D. Titley-Peloquin, Characterizing matrices that are consistent with given solutions, SIAM J. Matrix Anal. Appl., 30 (2008), pp. 1406-1420.

[4] S.-C. ChoI, Iterative Methods for Singular Linear Equations and Least-Squares Problems, Ph.D. thesis, Stanford University, Stanford, CA, 2006.

[5] G. Golub AND W. Kahan, Calculating the singular values and pseudo-inverse of a matrix, SIAM J. Numer. Anal., 2 (1965), pp. 205-224.

6] G. H. Golub and C. F. Van Loan, Matrix Computations, 3rd ed., The Johns Hopkins University Press, Baltimore, MD, 1996.

[7] J. F. GrCar, Optimal Sensitivity Analysis of Linear Least Squares, Technical report LBNL52434, Lawrence Berkeley National Laboratory, Berkeley, CA, 2003.

[8] M. GU, Backward perturbation bounds for linear least squares problems, SIAM J. Matrix Anal. Appl., 20 (1998), pp. 363-372.

[9] N. J. Higham, Accuracy and Stability of Numerical Algorithms, 2nd ed., SIAM, Philadelphia, 2002.

[10] The Matrix Market, available online at http://math.nist.gov/MatrixMarket.

[11] C. C. Paige And M. A. Saunders, Solution of sparse indefinite systems of linear equations, SIAM J. Numer. Anal., 12 (1975), pp. 617-629.

[12] C. C. Paige And M. A. SAunders, LSQR: An algorithm for sparse linear equations and sparse least squares, ACM Trans. Math. Software, 8 (1982), pp. 43-71.

[13] C. C. Paige And M. A. Saunders, Algorithm 583, LSQR: Sparse linear equations and sparse least squares problems, ACM Trans. Math. Software, 8 (1982), pp. 195-209.

[14] J. L. Rigal And J. Gaches, On the compatibility of a given solution with the data of a linear system, J. ACM, 14 (1967), pp. 543-548.

[15] G. W. SteWART, On the perturbation of pseudo-inverses, projections and linear least squares problems, SIAM Rev., 19 (1977), pp. 634-662.

[16] G. W. Stewart, Research, development, and LINPACK, in Mathematical Software III, J. R. Rice, ed., Academic Press, New York, 1977, pp. 1-14.

[17] Z. Strakoš And P. Tichy, On error estimation in the conjugate gradient method and why it works in finite precision computations, Electron. Trans. Numer. Anal., 13 (2002), pp. 5680.

[18] B. Waldén, R. Karlson, And J.-G. Sun, Optimal backward perturbation bounds for the linear least squares problem, Numer. Linear Algebra Appl., 2 (1995), pp. 271-286. 